\title{
Two-Dimensional Route Switching in Cognitive Radio Networks: A Game-Theoretical Framework
}

\author{
Qingkai Liang, Xinbing Wang, Xiaohua Tian, Fan Wu, Qian Zhang
}

\begin{abstract}
In Cognitive Radio Networks (CRNs), Secondary Users (SUs) can flexibly access Primary Users' (PUs') idle spectrum bands but such spectrum opportunities are dynamic due to PUs' uncertain activity patterns. In a multi-hop CRN consisting of SUs as relays, such spectrum dynamics will further cause the invalidity of pre-determined routes. In this paper, we investigate spectrum-mobility-incurred route-switching problems in both spatial and frequency domains for CRNs, where spatial switching determines which relays and links should be re-selected and frequency switching decides which channels ought to be re-assigned to the spatial routes. The proposed route-switching scheme not only avoids conflicts with PUs but also mitigates spectrum congestion. Meanwhile, tradeoffs between routing costs and channel switching costs are achieved. We further formulate the route-switching problem as the Route-Switching Game which is shown to be a potential game and has a pure Nash Equilibrium (NE). Accordingly, efficient algorithms for finding the NE and the $\epsilon-\mathrm{NE}$ are proposed. Then we extend the proposed game to the incomplete-information scenario and provide a method to compute the Bayesian NE. Finally, we prove that the price of stability of the proposed game has a deterministic upper bound.
\end{abstract}

Keywords-Cognitive Radio Networks, Game Theory, Routing, Spectrum Dynamics

\section{INTRODUCTION}

Cognitive Radio Networks (CRNs) have been proposed as a promising architecture for relieving spectrum shortages [1], where Secondary Users (SUs) can flexibly access Primary Users' (PUs') idle channels. Such Dynamic Spectrum Access (DSA) significantly improves spectrum utilization but brings new challenges to the design of CRNs at the same time, one of which is spectrum mobility.

In CRNs, PUs can reclaim their licensed channels at any time due to their high priority in occupying channels, and SUs must cease their transmission ${ }^{1}$ on those spectrum bands. Hence, from SUs' perspective, spectrum availability is dynamic due to PUs' uncertain channel reclamation behaviors, which further causes the aforementioned spectrum mobility.

In the context of multi-hop CRNs where SUs act as re-

A preliminary version of this work was published in Proceedings of ACM MobiHoc 2013 [4]. Q. Liang, X. Wang and X. Tian are with the Department of Electronic Engineering, Shanghai Jiao Tong University, China ( $\{$ victor856, xwang8, xtian $\} @$ sjtu.edu.cn). F. Wu is with the Department of Computer Science and Engineering, Shanghai Jiao Tong University, China (fwu@cs.sjtu.edu.cn). Q. Zhang is with the Department of Computer Science and Engineering, Hong Kong University of Science and Technology, Hong Kong (qianzh@cse.ust.hk).

${ }^{1}$ Such a method is also referred as the spectrum overlay mode. An alternative way is to ensure that the amount of generated interference to PUs is below a certain threshold, namely the spectrum underlay mode [9]. In this paper, we only consider the overlay mode. lays $^{2}$, spectrum mobility may further cause the break of preestablished routes of incoming data flows, since the unavailability of PUs' channels disables the transmission over some links on those routes. To avoid conflicts with PUs and resume routing, each flow initiator can either inform intermediate SU relays of switching their accessing channels or re-select a new spatial route ${ }^{3}$ where channels are not reclaimed. However, the following tradeoff implies that two-dimensional route switching (i.e., the combination of both channel switching and spatial route re-selection) is a better choice.

The advantage of channel switching is that it maintains the original optimal spatial route (e.g., a route with the fewest hops), which efficiently reduces routing costs, including transmission delay, energy consumption, etc. Unfortunately, frequent channel switching may cause significant switching costs such as switching delay, additional wear and tear, etc. By comparison, re-selecting a new spatial route can yield fewer switching costs. For instance, we can re-select a spatial route that only consists of links whose assigned channels are not reclaimed, which incurs zero switching cost. However, it may lead to additional routing costs at the same time (e.g., the new spatial route may consist of more hops). Consequently, there's a tradeoff between the two costs, which must be achieved by switching routes in both spatial and frequency domains.

Figure 1 shows a simple example that motivates the proposed two-dimensional route switching. The number next to each edge indicates the corresponding costs. Suppose a certain flow has source $A$ and destination $D$. At the beginning, all channels are available, so the optimal path is obviously $A \rightarrow E \rightarrow D$ with costs 2 (Figure 1 (a)). Now, suppose the channel used by link $(A, E)$ is reclaimed by PUs (Figure 1 (b)). Then we can choose to either switch the tuned channel on link $(A, E)$ to an idle one (say channel 6) or select a new route $A \rightarrow B \rightarrow C \rightarrow D$. If channel switching costs are 1 (Figure 1 (c)), then the former choice is preferred since the total costs would be 3 (additional switching cost 1 plus original routing cost 2). By comparison, the total costs are 4 if we choose the new route $A \rightarrow B \rightarrow C \rightarrow D$. However, if channel switching costs are 3 (Figure $1(\mathrm{~d})$ ), then the total costs incurred in the former case become 5, which implies that rerouting is a better choice. Hence, depending on specific contexts, we should flexibly choose between channel switching and rerouting.

\footnotetext{
${ }^{2}$ Since we focus on routing in the secondary network, we will use "CRN" and "secondary network" interchangeably in this paper.

${ }^{3}$ In the following, we will refer the selections of intermediate nodes and edges as spatial routes and the choices of channels used on the spatial routes as frequency routes.
} 


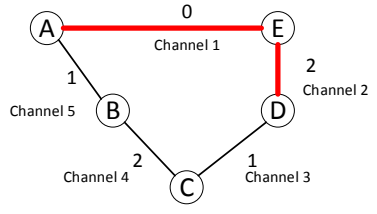

(a) original route and channel assignment

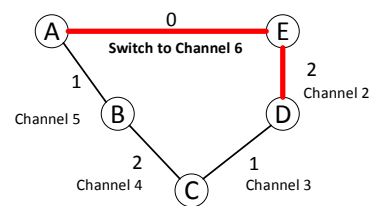

(c) strategy update when switching costs are 1

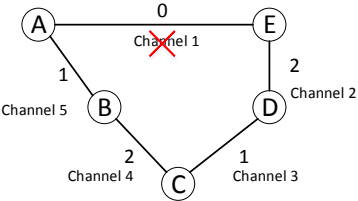

(b) channel 1 is reclaimed by PUs

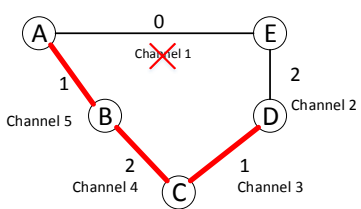

(d) strategy update when switching costs are 3

Fig. 1. An example of two-dimensional route switching.

In this paper, we propose Route-Switching Games to address the above spectrum-mobility-incurred route-switching problem in CRNs. The contributions of this paper include the following aspects.

- To our best knowledge, this paper is the first to investigate spectrum-mobility-incurred route-switching problems in CRNs. Accounting for selections in both spatial and frequency domains, our scheme not only avoids the conflicts with PUs but also mitigates spectrum congestion and achieves the tradeoff between routing and channel switching costs.

- We formulate the proposed problem as the RouteSwitching Game which is proved be a potential game. Efficient algorithms for finding the Nash Equilibrium (NE) and the $\epsilon-\mathrm{NE}$ are provided in this paper.

- We further study the game with incomplete information, where players' parameters are private. In such a scenario, a Bayesian NE is proved to exist and an algorithm for calculating the Bayesian NE is provided.

- We compare the NE of this game with socially optimal results in terms of social costs, namely the price of stability, which is upper-bounded by deterministic factors.

The remainder of this paper is organized as the following. We will first introduce the system model in section II. Next, in sections III and IV, Route-Switching Games with complete and incomplete information will be demonstrated, respectively. Then we will analyze the price of stability in section $\mathrm{V}$ with some additional discussions in section VI. Finally, simulation results, related works and conclusions will be given in sections VII, VIII and IX, respectively.

\section{NeTWORK MOdEL}

\section{A. Architecture of Multi-hop CRNs}

We consider a multi-hop CRN where multiple SUs act as routers for incoming data flows, and there're $C$ orthogonal and homogeneous channels accessible to SUs when they are not occupied by PUs (each channel is denoted by $j \in \mathcal{C}=$ $\{1,2, \cdots, C\})$. For the simplicity of analysis, we assume the entire secondary network lies in the same "collision domain" with $\mathrm{PUs}^{4}$, i.e., the perceived channel states (either busy or

\footnotetext{
${ }^{4}$ Note that our scheme can also be modified to incorporate the spatial diversity of PUs' spectrums in secondary networks.
}

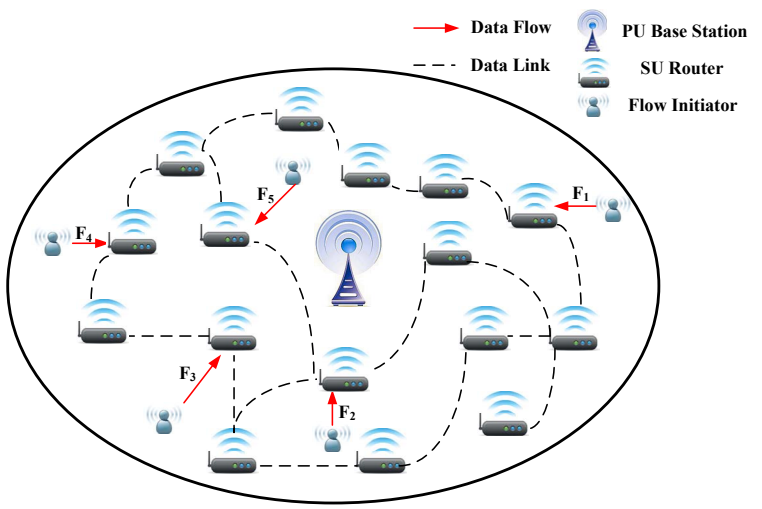

Fig. 2. An example of the multi-hop and multi-flow CRN. Note that the entire secondary network is within the transmission range of the PU base station, so the spectrum opportunities perceived at each SU are identical.

idle) at each SU are identical in the entire network. This assumption is valid for many geographically-centric secondary networks coexisting with powerful PU transceivers, like PU base stations in cellular networks, as is shown in Figure 2.

Formally, the entire secondary network can be characterized by a topological graph $G=(V, E)$. Here, $V$ is the set of nodes (SUs) and $E$ is the set of edges, where an edge exists between a pair of nodes $(u, v)$ iff they're within the transmission range of each other, so an edge corresponds with a data link. However, for a link to be able to sustain data transmission, it must be allocated a certain channel. As our focus is the route-switching problem, we suppose each link was formerly assigned a certain licensed channel (but these pre-assigned channels may be reclaimed by PUs and become unavailable now). Here, we denote matrix $\mathbf{A}$ the indication of pre-assigned channels on different links. Specifically, $A_{e, j}=1$ implies that channel $j$ was pre-assigned to link $e$ and $A_{e, j}=0$ otherwise.

\section{B. Flow Model}

Suppose there're $M$ concurrent data flows ${ }^{5}$ into the secondary network (each flow is denoted by $F_{k}, k \in \mathcal{M}=$ $\{1,2, \cdots, M\})$, and denote the source and destination of data flow $F_{k}$ by a pair $\left(S_{k}, D_{k}\right)$. For the efficiency and reliability of transmission, flow $F_{k}$ segments its data into many smaller packets, each with size $\mu_{k}$. We denote the flow rate of $F_{k}$ by $r_{k}$ and assume that those data flows are from different initiators, each hoping to minimize its own costs. Beside, we suppose that nodes in the secondary network will always honestly follow the routing plans developed by flow initiators. Cases where "malicious" secondary nodes exist are left for our future works.

\section{Spectrum Mobility and Route Switching}

When high-priority PUs reclaim their licensed channels, SUs must cease their transmission on those spectrum bands, which causes spectrum mobility. Here, we denote $\Gamma$ the set

\footnotetext{
${ }^{5} \mathrm{We}$ assume those data flows can last for a period of time like minutes or hours, which is particularly suitable for characterizing multimedia streaming, P2P downloading, etc.
} 
of channels that are currently unavailable to SUs due to PUs' reclamation. In practice, $\Gamma$ can be obtained by flow initiators without incurring significant overhead costs through our implementation (see section VI-B).

Unlike many earlier works that proposed statistical models to characterize PUs' reclaiming activities [9], [10], we do not predict PUs' behaviors, i.e., our scheme is reactive, since the precision of predictions still remains a major problem. Besides, route-switching schemes should provide routing reliability as much as possible, instead of probabilistic results, because the focus of the proposed mechanism is exactly to handle the negatives effects of spectrum uncertainty, which is the other reason why we don't choose proactive models.

As is mentioned in the introduction part, in face of spectrum mobility, routes must be switched in both spatial and frequency domains so as to avoid conflicts with PUs, mitigate congestion, and balance routing costs and channel switching costs (see section II-E for the formal definitions). Here, we use a 3-D matrix $\mathbf{X}$ to characterize the new selection of spatial routes and channel assignment, which is also the decision variable in the considered problem. Specifically, its element $X_{e, j}^{k}=1$ when link $e$ is included in the new spatial route of data flow $F_{k}$ and channel $j$ is re-assigned to this link ( $X_{e, j}^{k}=0$ otherwise).

\section{Interference Model and Constraints}

We use the protocol interference model [7], where the transmission in channel $j$ over link $e$ succeeds if all potential interferers in the interference neighborhood of link $e$ remain silent in channel $j$ for the transmission duration. Here, the interference neighborhood of link $e$, i.e., $I(e)$, is the set of links whose end nodes have interference links or data links incident on the end nodes of $e$. Further, when channel $j$ is perceived idle over link $e$, the contention window is activated and link $e$ will contend for the transmission opportunities with all interfering links in $I(e)$ (specifically, it's the transmitter on one end of link $e$ that executes the contention). This model resembles CSMA/CA in IEEE 802.11, based on an RTS-CTSData-ACK sequence.

Then we introduce the set of constraints in our model. First of all, any reclaimed channels cannot be assigned, i.e.,

$$
X_{e, j}^{k}=0, \forall j \in \Gamma, e \in E, k \in \mathcal{M} .
$$

Besides, we assume that any channel can only be assigned to at most one flow over the same link, considering the significant co-channel collisions and interference incurred on the same link. This yields the constraint

$$
\sum_{k \in \mathcal{M}} X_{e, j}^{k} \leq 1, \forall e \in E, j \in \mathcal{C} .
$$

Additionally, we also have the following radio constraint:

$$
\sum_{e \in E(v)} \sum_{k \in \mathcal{M}} \sum_{j \in \mathcal{C}} X_{e, j}^{k} \leq \alpha_{v}, \forall v \in V,
$$

where $E(v)$ is the set of edges incident on node $v$ and $\alpha_{v}$ is number of radios that node $v$ equips. This constraint shows that the number of channels to which a certain node (SU) tunes should not exceed its radio limitation. By the above three constraints, the feasible set $\mathcal{S}$ of this problem is defined to be the set of possible solutions that satisfy (1), (2) and (3).

\section{E. Cost Model}

We model the costs of each data flow by (i) routing costs incurred by relaying packets on the established route, and (ii) switching costs consumed to change the tuned channels over certain links.

1) Routing Costs: For flow $F_{k}$, its routing costs $R C_{k}$ are modeled by

$$
R C_{k}=D C_{k}+E C_{k},
$$

where $D C_{k}$ corresponds to the costs incurred by end-to-end delay from source $S_{k}$ to destination $D_{k}$, and $E C_{k}$ characterizes the costs resulting from energy consumption used for relaying the packets of $F_{k}$.

We first characterize delay costs. Under the interference model mentioned above, significant contention delay will be incurred if channels are congested since SUs must contend and wait for transmission opportunities. Note that there's no queuing delay in our model because constraint (2) implies different flows actually use different channels (thus different queues) at the same node. Hence, if we further neglect other minor delay like propagation delay, then contention delay can be regarded as a rough estimation of the overall one-hop delay.

As is typical of many random access protocols [24], [25], we make the following assumption: within the contention window, a certain link and all its interfering links have the same probability of wining the access to a certain channel $j \in \mathcal{C}$. Following the methods provided in [29]-[31], we can obtain an approximate expression for the expected contention delay within one hop, which characterizes the expected waiting time before flow $F_{k}$ gets the opportunity to transmit one packet in channel $j$ over link $e$ :

$$
\lambda_{e, j}=\delta_{e, j} \sum_{e^{\prime} \in I(e)} \sum_{k \in \mathcal{M}} X_{e^{\prime}, j}^{k} \omega_{k},
$$

where $\delta_{e, j}$ is a constant related to link $e$ and channel $j$. Without loss of generality, we can let $\delta_{e, j}=1$ in the rest of this paper, but our analysis carries over arbitrary values of $\delta_{e, j}$. Besides, in (4), we define $\omega_{k}=\mu_{k} / r_{k}$, i.e., the amount of time required by flow $F_{k}$ for transmitting one packet. The derivation of equation (4) is beyond the scope of this paper, so we omit it for brevity. The intuition behind equation (4) is explained as the following. $\sum_{k \in \mathcal{M}} X_{e^{\prime}, j}^{k} \omega_{k}$ in equation (4) represents the traffic demands (for transmission time) in channel $j$ over link $e^{\prime}$ imposed by all passing data flows, and thus equation (4) corresponds to the aggregate traffic demands in channel $j$ from the entire interference neighborhood of link $e$. Generally speaking, $\lambda_{e, j}$ reflects the congestion level of channel $j$ perceived over link $e$, so delay costs can also be interpreted as the congestion costs in our model. As a result, although equation (4) is only an approximation to one-hop delay, it precisely reflects the root of delay, namely network congestion. In this sense, it is as desired as the precise delay expression which may be difficult to characterize in reality.

For denoting simplicity, we introduce a 0-1 indicator $\theta_{e, e^{\prime}}$ to imply the interference relationship. Specifically, $\theta_{e, e^{\prime}}=1$ indicates that link $e^{\prime}$ is in the interference neighborhood of $e$. Note that $\theta_{e, e}=0$ for any $e \in E$ and we consider mutual interference, which means that $\theta_{e, e^{\prime}}=\theta_{e^{\prime}, e}$. Besides, interference 
caused by one's own transmission over other interfering links is neglected for the tractability of analysis, since recent literatures (e.g., [17], [18]) have suggested such interference can be mitigated significantly by exploiting the self-interference cancellation technology in relay systems. Therefore, we can rewrite the expected one-hop delay perceived by $F_{k}$ when it is transmitting in channel $j$ over link $e$ by:

$$
\lambda_{e, j}^{k}=\sum_{e^{\prime} \in E} \sum_{k^{\prime} \in \mathcal{M}_{k}} X_{e^{\prime}, j}^{k^{\prime}} \omega_{k^{\prime}} \theta_{e, e^{\prime}}
$$

where $\mathcal{M}_{k}=\mathcal{M} \backslash\{k\}$. Further, we can characterize the expected end-to-end delay as the sum of hop-by-hop delay. Then the delay costs of flow $F_{k}$ are given by

$$
D C_{k}=P_{d} \sum_{e \in E} \sum_{j \in \mathcal{C}} X_{e, j}^{k} \lambda_{e, j}^{k},
$$

which is proportional to the expected end-to-end delay. Here, $P_{d}$ is a constant reflecting the revenue lost per unit of delay.

Next, we consider energy costs which characterize power consumption used for relaying packets. Under our interference model, when one SU transmits in channel $j$ over link $e$, other SUs within $I(e)$ must remain silent in channel $j$, so the SINR perceived at each SU receiver is merely dependent on transmission power, intrinsic channel quality and geographical conditions (e.g., path loss). Noticing the above fact, we model power consumption as a general function $\varphi_{e, j}\left(r_{k}\right)$ (and $\varphi_{e, j}^{k}$ for short), which neatly captures the influence of flow rates, channel quality and geographical conditions on power consumption. Note that $\varphi_{e, j}^{k}$ can be of different forms, depending on the features of wireless networks ${ }^{6}$. Then, the energy costs of flow $F_{k}$ are shown as:

$$
E C_{k}=P_{e} \sum_{e \in E} \sum_{j \in \mathcal{C}} X_{e, j}^{k} \varphi_{e, j}^{k} .
$$

Similarly, $P_{e}$ is a constant reflecting the revenue lost per unit of power consumption.

2) Switching Costs: Different from the above routing costs, switching costs characterize the potential expense used for channel handoff. Here, we use $\gamma$ to indicate the overall revenue lost per channel switching, which may include (i) additional energy consumption used for sensing and establishing new connections, (ii) switching delay, (iii) increased wear and tear during channel reconfiguration, etc. For example, in terms of switching delay, many practical mobile systems like Qualcomm's MediaFLO, show switching delay around 1.5s [26]. Note that $\gamma$ includes the costs of both $\mathrm{ON} \rightarrow \mathrm{OFF}$ switching (tearing down the old channel connection) and $\mathrm{OFF} \rightarrow \mathrm{ON}$ switching (establishing the new channel connection), so the two types of switching costs can be seen as being incurred altogether in either switching scenario. In this paper, we assume the overall switching costs $\gamma$ are incurred only in the $\mathrm{OFF} \rightarrow \mathrm{ON}$ transition.

\footnotetext{
${ }^{6}$ For example, a possible expression of $\varphi_{e, j}^{k}$ is based on Shannon Formula in the presence of AWGN, i.e., $r_{k}=W_{j} \log \left(1+\varphi_{e, j}^{k} d^{-\alpha} / \sigma_{e, j}^{2}\right)$, where $W_{j}$ is the bandwidth of channel $j, \sigma_{e, j}^{2}$ is the variance of AWGN in channel $j$ over link $e, d$ is the distance between the transmitter and the receiver, $\alpha$ is the attenuation coefficient, and $\varphi_{e, j}^{k}$ is the corresponding transmission power. In this case, power consumption $\varphi_{e, j}^{k}$ can be readily obtained.
}

Therefore, the total channel switching costs associated with $F_{k}$ 's strategy are

$$
S C_{k}=\sum_{e \in E} \sum_{j \in \mathcal{C}} X_{e, j}^{k} \gamma_{e, j},
$$

where we define $\gamma_{e, j}=\left(1-A_{e, j}\right) \gamma$. Corresponding to the above discussion, equation (8) implies that only when $A_{e, j}=$ 0 and $X_{e, j}^{k}=1$ (i.e., link $e$ did not use channel $j$ in the past, but now this channel is allocated to $e$ according to $F_{k}$ 's strategy), switching costs are incurred.

So far, we have defined two types of major costs in the network: routing costs (i.e., delay costs plus energy costs) and switching costs. In reality, the two types of costs conflict with each other and cannot be simultaneously minimized (see section VI-A for the detailed discussion). Hence, when designing the overall cost function, we aim at achieving the tradeoff between the two types of costs. Specifically, we model flow $F_{k}$ 's overall cost function as:

$$
T C_{k}=\Omega_{R} R C_{k}+\Omega_{S} S C_{k} .
$$

Here, $\Omega_{R}$ and $\Omega_{S}$ are two non-negative system parameters characterizing the relative importance of routing costs and switching costs, respectively. For example, if nodes in the secondary network are energy-constrained, we tend to set $\Omega_{R}$ to be a large value while keeping $\Omega_{S}$ small; if the CRN is delay-tolerant and energy-abundant but has a low tolerance for channel switching, we prefer a small $\Omega_{R}$ and a large $\Omega_{S}$. In fact, the two parameters provide us with the flexibility of balancing routing costs and switching costs. Particularly, if $\Omega_{R}>0$ and $\Omega_{S}=0$, routing costs are minimized; if $\Omega_{R}=0$ and $\Omega_{S}>0$, switching costs are minimized; if $\Omega_{R}>0$ and $\Omega_{S}>0$, a tradeoff (of a certain degree) is obtained.

Additionally, without loss of generality, we assume that $P_{d}=P_{e}=1$ in the rest of this paper. With trivial changes, our analysis can be easily applied to the case where these parameters take arbitrarily feasible values.

\section{Route-Switching GAmes With Complete INFORMATION}

From equation (5), we can obviously observe that a certain flow's delay costs are also dependent on others' routeswitching strategies, so we formulate the above problem as Routing-Switching Games, where players (i.e., flow initiators) distributively and selfishly switch their two-dimensional routes in face of spectrum mobility, aiming at minimizing their own overall cost functions.

\section{A. Game Formulation}

Under complete information, each player's information (i.e., data rate $r_{k}$ and packet size $\mu_{k}, \forall k \in \mathcal{M}$ ) is known to others. We can use a tuple $\mathcal{G}=\{G, \mathbf{A}, \Gamma, r, \mu, T C, S\}$ to denote the Route-Switching Game with complete information. Here, the meanings of $G, \mathbf{A}$ and $\Gamma$ have been explained in section II. $r=\left\{r_{1}, \cdots, r_{M}\right\}$ and $\mu=\left\{\mu_{1}, \cdots, \mu_{M}\right\}$ are publiclyknown parameter vectors of flows. $T C$ is the set of players' cost functions, shown in (9). $S=\{(e, j) \mid e \in E, j \in \mathcal{C}\}$ is the two-dimensional strategy space. In this paper, we consider 
the symmetric game where all players have the same strategy space. Further, we denote $s=\left\{s_{1}, \cdots, s_{M}\right\}$ the strategy profile, where $s_{k}=\left\{(e, j) \in S \mid X_{e, j}^{k}=1\right\}$ is flow $F_{k}$ 's strategy ${ }^{7}$. Note that the different kinds of costs of flow $F_{k}$ as well as the value of $\lambda_{e, j}^{k}$ depend on the strategy profile $s$, so we denote them by $R C_{k}(s), D C_{k}(s), E C_{k}(s), S C_{k}(s)$, $T C_{k}(s)$ and $\lambda_{e, j}^{k}(s)$, respectively ${ }^{8}$.

In addition, it's worthy mentioning that the above formulation does not impose any constraints on the connectivity of switched routes but such an omission won't influence any of the following analytical results. Instead, we guarantee the connectivity through our algorithm implementation (see Theorem 3 in section III-C).

Finally in this subsection, we give the definition of the Nash Equilibrium $^{9}$, which will be frequently discussed.

Definition 1 (Nash Equilibrium, NE): A strategy profile $s^{*}=\left(s_{1}^{*}, s_{2}^{*}, \cdots, s_{M}^{*}\right)$ is a Nash Equilibrium if for any player $F_{k}(\forall k \in \mathcal{M})$ and its any strategy $s_{k} \subseteq S$,

$$
T C_{k}\left(s_{k}^{*} ; s_{-k}^{*}\right) \leq T C_{k}\left(s_{k} ; s_{-k}^{*}\right),
$$

where $s_{-k}^{*}$ is the strategy profile $s^{*}$ excluding $s_{k}^{*}$. By definition, no player can reduce its own costs by unilaterally changing the strategy at the equilibrium.

\section{B. Potential Game}

The potential game [33] is a relatively new game-theoretical model which can characterize a wide range of games, including the classical congestion game [32]. It has already demonstrated its importance through many successful applications in practical problems like spatial spectrum access [19] [20], gateway selections [21], etc.

In the rest of this subsection, we will briefly introduce the concept of the potential game and its properties, which will be further exploited in this paper.

Definition 2 (Potential Game): A game is referred as the potential game if and only if there exists a potential function in the game.

Definition 3 (Potential Function): A function $\Phi(s)$ is the potential function for the minimum game $^{10} \mathcal{G}$ if for any strategy profile $s$, any player $F_{k}(\forall k \in \mathcal{M})$ and its any two strategies $s_{k}, s_{k}^{\prime} \subseteq S$

$$
\begin{aligned}
& T C_{k}\left(s_{k}^{\prime} ; s_{-k}\right)-T C_{k}\left(s_{k} ; s_{-k}\right)<0 \\
\Rightarrow & \Phi\left(s_{k}^{\prime} ; s_{-k}\right)-\Phi\left(s_{k} ; s_{-k}\right)<0 .
\end{aligned}
$$

That is, if any player can unilaterally reduce its costs, the value of the potential function will also be reduced. Potential games have many ideal properties, and we mainly use three of them.

\footnotetext{
${ }^{7}$ In this paper, each player's (say flow $F_{k}$ 's) strategy can be expressed in two forms: $s_{k}$ and the corresponding $0-1$ indication $X_{e, j}^{k}(e \in E, j \in \mathcal{C})$. The two forms are equivalent and will be used interchangeably in the following. Note that $(e, j) \in s_{k}$ if and only if $X_{e, j}^{k}=1$.

${ }^{8}$ To be more specific, $D C_{k}, R C_{k}$ and $T C_{k}$ are dependent on the entire strategy profile $s ; \lambda_{e, j}^{k}$ is dependent on the entire profile expect $s_{k} ; E C_{k}$ and $S C_{k}$ are only relevant to flow $F_{k}$ 's own strategy $s_{k}$. For denoting simplicity, they are uniformly expressed as the function of $s$.

${ }^{9}$ We only consider the pure NE throughout this paper.

${ }^{10} \mathrm{~A}$ game is a minimum game if players tend to minimize their cost functions.
}

Property 1: Every finite potential game ${ }^{11}$ has at least one pure Nash Equilibrium.

From the definition of the potential function, we can observe that the minimum of the potential function corresponds to a pure $\mathrm{NE}$ in the minimum game. That is, no player can unilaterally decrease its costs at the minimum of the potential function, otherwise the reduction of this player's costs will also lead to the reduction of the potential function, violating the definition of the minimum.

Property 2: Every finite potential game has the Finite Improvement Property (FIP).

The meaning of FIP is as the following. Initially, each player can randomly select its own strategy. Then every player rotates to improve its strategy by reducing the potential function with others' strategies fixed. After finite improvement steps, the potential function will reach the minimum, and thus an NE is derived. FIP actually provides us with a feasible method to compute an NE in the potential game, which will be further utilized in section III-C.

Property 3: Every finite potential game has at least one pure $\epsilon$-Nash Equilibrium.

We temporarily skip the explanation of Property 3. Further discussions will be given in section III-D.

Proofs to the three properties can be found in [33].

\section{Existence and Computation of the $N E$}

In this subsection, we will first show that the proposed Route-Switching Game is essentially a potential game. Then an algorithm for computing the NE will be provided.

Theorem 1: Under complete information, the proposed Route-Switching Game is a finite potential game which has the following potential function:

$$
\Phi(s)=\sum_{k \in \mathcal{M}} \omega_{k}\left[\Omega_{R} D C_{k}(s)+2 \Omega_{R} E C_{k}(s)+2 \Omega_{S} S C_{k}(s)\right]
$$

Proof: It's obvious that the proposed Route-Switching Game is finite, and we only prove that (10) is a potential function. Consider an improvement from strategy profile $s$ to $q$. The 0-1 strategy indications corresponding to $s$ and $q$ are $X_{e, j}^{k}$ and $X_{e, j}^{\prime k}$, respectively. The only difference between $s$ and $q$ is that player $F_{k}$ improves its strategy from $s_{k}$ to $q_{k}$, which implies $s_{-k}=q_{-k}$ and

$$
T C_{k}(s)>T C_{k}(q)
$$

At the same time, we define:

$$
\zeta_{e, j}^{k}(s):=\omega_{k}\left[\Omega_{R} \lambda_{e, j}^{k}(s)+2 \Omega_{R} \varphi_{e, j}^{k}+2 \Omega_{S} \gamma_{e, j}\right]
$$

\footnotetext{
${ }^{11} \mathrm{~A}$ game is said to be finite when each player has a finite number of options and the number of players is also finite.
} 
Thus we can derive

$$
\begin{aligned}
& \Phi(s)-\Phi(q) \\
& =\sum_{k^{\prime} \in \mathcal{M}} \sum_{(e, j) \in s_{k^{\prime}}} \zeta_{e, j}^{k^{\prime}}(s)-\sum_{k^{\prime} \in \mathcal{M}} \sum_{(e, j) \in q_{k^{\prime}}} \zeta_{e, j}^{k^{\prime}}(q) \\
= & \left.\sum_{(e, j) \in s_{k}} \zeta_{e, j}^{k}(s)-\sum_{(e, j) \in q_{k}} \zeta_{e, j}^{k}(q)\right) \\
& +\left(\sum_{k^{\prime} \in \mathcal{M}_{k}} \sum_{(e, j) \in s_{k^{\prime}}} \zeta_{e, j}^{k^{\prime}}(s)-\sum_{k^{\prime} \in \mathcal{M}_{k}} \sum_{(e, j) \in q_{k^{\prime}}} \zeta_{e, j}^{k^{\prime}}(q)\right) .
\end{aligned}
$$

For the first term in the above equation, we can further write out its explicit expression

$$
\begin{aligned}
& \sum_{(e, j) \in s_{k}} \zeta_{e, j}^{k}(s)-\sum_{(e, j) \in q_{k}} \zeta_{e, j}^{k}(q) \\
= & \sum_{e \in E} \sum_{j \in \mathcal{C}} X_{e, j}^{k} \omega_{k}\left[\Omega_{R} \lambda_{e, j}^{k}(s)+2 \Omega_{R} \varphi_{e, j}^{k}+2 \Omega_{S} \gamma_{e, j}\right] \\
& -\sum_{e \in E} \sum_{j \in \mathcal{C}} X_{e, j}^{\prime k} \omega_{k}\left[\Omega_{R} \lambda_{e, j}^{k}(q)+2 \Omega_{R} \varphi_{e, j}^{k}+2 \Omega_{S} \gamma_{e, j}\right] .
\end{aligned}
$$

For the second term in (11), we first notice that $s_{k^{\prime}}=q_{k^{\prime}}$ for any $k^{\prime} \in \mathcal{M}_{k}$, i.e.,

$$
X_{e, j}^{k^{\prime}}=X_{e, j}^{\prime k^{\prime}}, \forall k^{\prime} \in \mathcal{M}_{k}, e \in E, j \in \mathcal{C} .
$$

Then the second term can be equivalently written as

$$
\begin{aligned}
& \sum_{k^{\prime} \in \mathcal{M}_{k}} \sum_{e \in E} \sum_{j \in \mathcal{C}} X_{e, j}^{k^{\prime}} \zeta_{e, j}^{k^{\prime}}(s)-\sum_{k^{\prime} \in \mathcal{M}_{k}} \sum_{e \in E} \sum_{j \in \mathcal{C}} X_{e, j}^{k^{\prime}} \zeta_{e, j}^{k^{\prime}}(q) \\
= & \sum_{k^{\prime} \in \mathcal{M}_{k}} \sum_{e \in E} \sum_{j \in \mathcal{C}} X_{e, j}^{k^{\prime}}\left[\zeta_{e, j}^{k^{\prime}}(s)-\zeta_{e, j}^{k^{\prime}}(q)\right] \\
= & \sum_{k^{\prime} \in \mathcal{M}_{k}} \sum_{e \in E} \sum_{j \in \mathcal{C}} X_{e, j}^{k^{\prime}} \omega_{k^{\prime}} \Omega_{R}\left[\lambda_{e, j}^{k^{\prime}}(s)-\lambda_{e, j}^{k^{\prime}}(q)\right] \\
= & \sum_{k^{\prime} \in \mathcal{M}_{k}} \sum_{j \in \mathcal{C}} \sum_{e \in E} \sum_{e^{\prime} \in E} X_{e, j}^{k^{\prime}} \omega_{k^{\prime}} \omega_{k} \theta_{e, e^{\prime}} \Omega_{R}\left(X_{e^{\prime}, j}^{k}-X_{e^{\prime}, j}^{\prime k}\right) .
\end{aligned}
$$

Note that the derivation of (14) exploits the fact that

$$
\begin{array}{r}
\lambda_{e, j}^{k^{\prime}}(s)-\lambda_{e, j}^{k^{\prime}}(q)=\sum_{e^{\prime} \in E} \omega_{k} \theta_{e, e^{\prime}}\left(X_{e^{\prime}, j}^{k}-X_{e^{\prime}, j}^{\prime k}\right), \\
\forall k^{\prime} \in \mathcal{M}_{k}, e \in E, j \in \mathcal{C},
\end{array}
$$

since only $F_{k}$ 's strategy changes while others' strategies remain the same in the strategy profiles $s$ and $q$.

Interchanging the role of $e$ and $e^{\prime}$ together with (13) and the assumption $\theta_{e, e^{\prime}}=\theta_{e^{\prime}, e}$, we can rewrite equation (14) as

$$
\begin{aligned}
\Omega_{R} & \left(\sum_{e \in E} \sum_{j \in \mathcal{C}} X_{e, j}^{k} \omega_{k} \sum_{k^{\prime} \in \mathcal{M}_{k}} \sum_{e^{\prime} \in E} X_{e^{\prime}, j}^{k^{\prime}} \omega_{k^{\prime}} \theta_{e, e^{\prime}}\right. \\
& \left.-\sum_{e \in E} \sum_{j \in \mathcal{C}} X_{e, j}^{\prime k} \omega_{k} \sum_{k^{\prime} \in \mathcal{M}_{k}} \sum_{e^{\prime} \in E} X_{e^{\prime}, j}^{\prime k^{\prime}} \omega_{k^{\prime}} \theta_{e, e^{\prime}}\right) \\
=\Omega_{R} & \left(\sum_{e \in E} \sum_{j \in \mathcal{C}} X_{e, j}^{k} \omega_{k} \lambda_{e, j}^{k}(s)-\sum_{e \in E} \sum_{j \in \mathcal{C}} X_{e, j}^{\prime k} \omega_{k} \lambda_{e, j}^{k}(q)\right) .
\end{aligned}
$$

By summing (12) and (15), we finally obtain that

$$
\begin{aligned}
& \Phi(s)-\Phi(q) \\
= & \sum_{e \in E} \sum_{j \in \mathcal{C}} X_{e, j}^{k} \omega_{k}\left[2 \Omega_{R} \lambda_{e, j}^{k}(s)+2 \Omega_{R} \varphi_{e, j}^{k}+2 \Omega_{S} \gamma_{e, j}\right] \\
& -\sum_{e \in E} \sum_{j \in \mathcal{C}} X_{e, j}^{\prime k} \omega_{k}\left[2 \Omega_{R} \lambda_{e, j}^{k}(q)+2 \Omega_{R} \varphi_{e, j}^{k}+2 \Omega_{S} \gamma_{e, j}\right] \\
= & 2 \omega_{k}\left[T C_{k}(s)-T C_{k}(q)\right]>0 .
\end{aligned}
$$

Hence, we have proved that (10) is a potential function of the proposed game.

According to Theorem 1 and Property 1 of general potential games, we can immediately conclude the following result.

Theorem 2: There exists a Nash Equilibrium in the RouteSwitching Game with complete information, and this NE minimizes the potential function in (10).

Next, we design an algorithm to reach the NE in Theorem 2, shown as Algorithm 1. Note that each player locally runs this algorithm to determine its own best strategy at the NE, by simulating other players' possible actions (step 3 to step 18). This is also referred as the Fictitious Play Process in game theory [33]. The accuracy of Fictitious Play is dependent on the degree of known information about other players. Under complete information, every player's parameters are precisely known by others, so the Fictitious Play Process will converge to the real play process. However, Fictitious Play will deviate from the reality under incomplete information (but it still converges), as will be further demonstrated in section IV. Essentially, Algorithm 1 is an iterative algorithm following FIP. Its major part is the strategy improvement (or update), which is done by first converting the reduction of the potential function into finding the shortest path in an undirected graph and then applying the well-known Dijsktra Algorithm to find such a path. Step 9 in Algorithm 1 handles the three constraints mentioned in section II-D, where $(e, j) \in \Lambda$ if letting $X_{e, j}^{k}=1$ will violate any of the three constraints under the strategy profile in that loop. Variable $m$ in the algorithm acts like a counter recording the consecutive times for which players cannot reduce the value of the potential function, and the stop condition (step 18) indicates that all $M$ players cannot reduce the potential function anymore, where the minimum point (i.e., the NE) is reached. Note that a player's strategy is updated only when it can reduce the potential function (steps 12 and 13) otherwise its previous strategy remains. Besides, the expression of $W_{(e, j)}$ in step 7 when $F_{k}$ is improving its strategy is given by

$$
W_{(e, j)}=2 \omega_{k}\left[\Omega_{R} \lambda_{e, j}^{k}\left(s_{-k}\right)+\Omega_{R} \varphi_{e, j}^{k}+\Omega_{S} \gamma_{e, j}\right],
$$

where $s_{-k}$ is the strategy profile of other flows obtained after the previous iteration. Note that although $\lambda_{e, j}^{k}$ is usually written as a function the entire strategy profile $s$ but it actually only depends on $s_{-k}$. Here, we explicitly write $\lambda_{e, j}^{k}$ as a function of $s_{-k}$ to emphasize that only flow $F_{k}$ is updating its strategy while others' actions are fixed. The correctness of (17) and Algorithm 1 is shown in the proof to Theorem 3.

Theorem 3: Each improvement step (i.e., each iteration) in Algorithm 1 can reduce the potential function to the maximum 


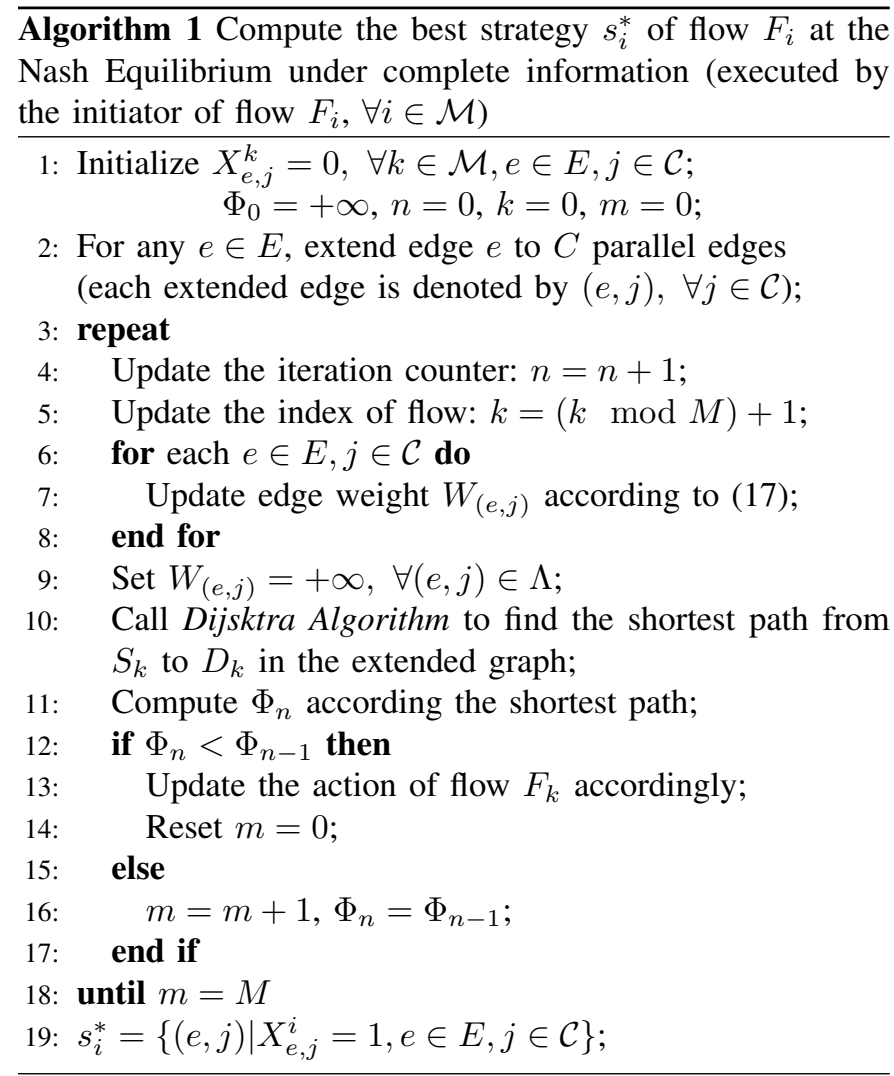

extent and guarantee route connectivity in polynomial time with the time complexity $O\left(|E| M+|V|^{2}\right)$.

Proof: Suppose $F_{k}$ is updating its strategy in the Fictitious Play Process shown in Algorithm 1. Then we have

$$
\Phi(s)=\sum_{k^{\prime} \in \mathcal{M}_{k}} \sum_{e \in E} \sum_{j \in \mathcal{C}} X_{e, j}^{k^{\prime}} \zeta_{e, j}^{k^{\prime}}(s)+\sum_{e \in E} \sum_{j \in \mathcal{C}} X_{e, j}^{k} \zeta_{e, j}^{k}(s),
$$

where

$$
\zeta_{e, j}^{k}(s):=\omega_{k}\left[\Omega_{R} \lambda_{e, j}^{k}\left(s_{-k}\right)+2 \Omega_{R} \varphi_{e, j}^{k}+2 \Omega_{S} \gamma_{e, j}\right] .
$$

Here, $s_{-k}$ (or equivalently, $X_{e, j}^{k^{\prime}}, \forall k^{\prime} \in \mathcal{M}_{k}, e \in E, j \in \mathcal{C}$ ) is the strategy profile of other flows obtained in the previous iteration, which is fixed when player $F_{k}$ is updating its strategy. Hence reducing $\Phi(s)$ is equivalent to reducing

$$
\begin{aligned}
\Phi^{\prime}(s)= & \sum_{k^{\prime} \in \mathcal{M}_{k}} \sum_{e \in E} \sum_{j \in \mathcal{C}} X_{e, j}^{k^{\prime}} \omega_{k^{\prime}}\left[\Omega_{R} \sum_{e^{\prime} \in E} X_{e^{\prime}, j}^{k} \omega_{k} \theta_{e, e^{\prime}}\right] \\
& +\sum_{e \in E} \sum_{j \in \mathcal{C}} X_{e, j}^{k} \zeta_{e, j}^{k}(s)
\end{aligned}
$$

For the first term in $\Phi^{\prime}(s)$,

$$
\begin{aligned}
& \sum_{k^{\prime} \in \mathcal{M}_{k}} \sum_{e \in E} \sum_{j \in \mathcal{C}} X_{e, j}^{k^{\prime}} \omega_{k^{\prime}}\left[\Omega_{R} \sum_{e^{\prime} \in E} X_{e^{\prime}, j}^{k} \omega_{k} \theta_{e, e^{\prime}}\right] \\
= & \Omega_{R} \sum_{e \in E} \sum_{j \in \mathcal{C}} \sum_{e^{\prime} \in E} X_{e^{\prime}, j}^{k} \sum_{k^{\prime} \in \mathcal{M}_{k}} X_{e, j}^{k^{\prime}} \omega_{k^{\prime}} \omega_{k} \theta_{e, e^{\prime}} \\
= & \Omega_{R} \sum_{e \in E} \sum_{j \in \mathcal{C}} X_{e, j}^{k} \sum_{e^{\prime} \in E} \sum_{k^{\prime} \in \mathcal{M}_{k}} X_{e^{\prime}, j}^{k^{\prime}} \omega_{k^{\prime}} \omega_{k} \theta_{e, e^{\prime}} \\
= & \sum_{e \in E} \sum_{j \in \mathcal{C}} X_{e, j}^{k} \omega_{k} \Omega_{R} \lambda_{e, j}^{k}\left(s_{-k}\right),
\end{aligned}
$$

Note that we interchange the role of $e$ and $e^{\prime}$ and exploit the fact that $\theta_{e, e^{\prime}}=\theta_{e^{\prime}, e}$ in the second equation. Hence, $\Phi^{\prime}(s)$ can be written as

$$
\Phi^{\prime}(s)=\sum_{e \in E} \sum_{j \in \mathcal{C}} X_{e, j}^{k} 2 \omega_{k}\left[\Omega_{R} \lambda_{e, j}^{k}\left(s_{-k}\right)+\Omega_{R} \varphi_{e, j}^{k}+\Omega_{S} \gamma_{e, j}\right] .
$$

Therefore, when $F_{k}$ is updating its strategy, if we set the weight of edge $(e, j)$ in the extended graph to be

$$
W_{(e, j)}=2 \omega_{k}\left[\Omega_{R} \lambda_{e, j}^{k}\left(s_{-k}\right)+\Omega_{R} \varphi_{e, j}^{k}+\Omega_{S} \gamma_{e, j}\right],
$$

then finding the shortest path in the extended graph will be equivalent to reducing $\Phi^{\prime}(s)$ to the maximum extent, which further reduces the potential function $\Phi(s)$ to the maximum extent. Since weight $W_{(e, j)} \geq 0$, then Dijsktra Algorithm can be applied to find the shortest path in the extended graph. Besides, route connectivity is guaranteed by the property of Dijsktra Algorithm.

In terms of the time complexity, we investigate how it scales with the number of players $(M)$ and the network scale $(|E|$ and $|V|)$. To compute $W_{(e, j)}$, we can first calculate and store the value of $\lambda_{e, j}^{k}$ at the end of the current iteration such that it can be readily used in the next iteration, which amounts to $O(|E| M)$ time in each iteration. With the value of $\lambda_{e, j}^{k}$, computing $W_{(e, j)}$ only needs $O(1)$ time for every $e \in E$ and $j \in \mathcal{C}$. Thus, setting weight $W_{(e, j)}$ on the extended graph will consume $O(|E|)$ time (note that $C$ can be regarded as a constant here) in each iteration and Dijsktra Algorithm is of $O\left(|V|^{2}\right)$. Then the overall time complexity of each iteration is $O\left(|E| M+|V|^{2}\right)$.

\section{D. $\epsilon$-Nash Equilibrium}

Algorithm 1 provides us with a method to compute the exact NE, where no players can reduce their own costs by unilaterally deviating from the NE. Unfortunately, Algorithm 1 is not guaranteed to reach the minimum of the potential function in polynomial time, even though simulation results show that the convergence is very fast (see section VII). Alternatively, we can obtain an approximate $N E$ or $\epsilon-N E$ in polynomial time, by modifying Algorithm 1. Firstly, we give the formal definition of the $\epsilon$-Nash Equilibrium.

Definition 4 ( $\epsilon$-Nash Equilibrium): A strategy profile $s^{*}=$ $\left(s_{1}^{*}, s_{2}^{*}, \cdots, s_{M}^{*}\right)$ is an $\epsilon$-Nash Equilibrium if for any player $F_{k}(\forall k \in \mathcal{M})$ and its any strategy $s_{k} \subseteq S$,

$$
T C_{k}\left(s_{k}^{*} ; s_{-k}^{*}\right) \leq T C_{k}\left(s_{k} ; s_{-k}^{*}\right)+\epsilon .
$$

The above definition implies that no player can reduce its costs by $\epsilon$ if it unilaterally violates the $\epsilon$-NE. Particularly, when $\epsilon=0$, the $\epsilon$-NE becomes the exact NE.

As a corollary of Property 3 of general potential games, we have the following theorem.

Theorem 4: Under complete information, every RouteSwitching Game has a unique $\epsilon$-Nash Equilibrium.

To compute the $\epsilon$-Nash Equilibrium, we only need to slightly modify Algorithm 1 by setting the condition in step 12 to be " $\Phi_{n}<\Phi_{n-1}-\epsilon$ ". By such modification, we can conclude Theorem 5 . 
Theorem 5: The computation of the $\epsilon$-Nash Equilibrium can terminate in $O\left(\frac{M^{2}|E||V|}{\epsilon}\right)$ iterations.

Proof: It's obvious that

$$
\lambda_{e, j}^{k}(s)=\sum_{e^{\prime} \in E} \sum_{k^{\prime} \in \mathcal{M}_{k}} X_{e^{\prime}, j}^{k^{\prime}} \omega_{k^{\prime}} \theta_{e, e^{\prime}} \leq|E| M \max _{k^{\prime} \in \mathcal{M}} \omega_{k^{\prime}} .
$$

Define $U:=\max _{k^{\prime}} \omega_{k^{\prime}}, Q:=\max _{k^{\prime}, e, j} \varphi_{e, j}^{k^{\prime}}$, and $W:=$ $\max _{v} \alpha_{v}$, where $\alpha_{v}$ is the number of radios equipped by node $v$. Then we have

$$
\begin{aligned}
\Phi(s) & =\sum_{k \in \mathcal{M}} \sum_{(e, j) \in s_{k}} \omega_{k}\left[\Omega_{R} \lambda_{e, j}^{k}(s)+2 \Omega_{R} \varphi_{e, j}^{k}+2 \Omega_{S} \gamma_{e, j}\right] \\
& \leq U\left(\Omega_{R}|E| M U+2 \Omega_{R} Q+2 \Omega_{S} \gamma\right) \sum_{e \in E} \sum_{j \in \mathcal{C}} \sum_{k \in \mathcal{M}} X_{e, j}^{k} \\
& \leq U W|V|\left(\Omega_{R}|E| M U+2 \Omega_{R} Q+2 \Omega_{S} \gamma\right),
\end{aligned}
$$

where the last inequality is according to (3). In the modified version of Algorithm 1, the value of $\Phi(s)$ will be reduced by at least $\epsilon$ after every $M$ improvement steps otherwise the algorithm will stop. Hence, noticing that $\Phi(s) \geq 0$, we can conclude that the maximum number of improvement steps will be upper-bounded by

$$
\frac{M \Phi(s)}{\epsilon} \leq \frac{M U W|V|\left(\Omega_{R}|E| M U+2 \Omega_{R} Q+2 \Omega_{S} \gamma\right)}{\epsilon}
$$

That is, the computation of the $\epsilon$-Nash Equilibrium can terminate in $O\left(\frac{M^{2}|E||V|}{\epsilon}\right)$ iterations.

\section{Route-Switching Games with Incomplete INFORMATION}

In the above sections, we assume that all players have the exact information about others. However, obtaining exact parameters about other concurrent flows could be very difficult in practice. As is often the case, obtaining statistical information is much easier. In this section, we will extend our scheme to the incomplete-information scenario, where players' exact information is hidden while their statistics is known.

The proposed game with incomplete information can be indicated by the tuple $\mathcal{G}=\{G, A, \Gamma, S, T C, \mathbf{T}, \mathbf{p}\}$. The slight differences between this definition and that of the completeinformation game lie in two aspects. Firstly, we introduce a type space $\mathbf{T}=\mathbf{T}_{\mathbf{1}} \times \cdots \times \mathbf{T}_{\mathbf{M}}$ to indicate the possible rates and packet sizes of data flows in the incomplete-information game, where $\mathbf{T}_{\mathbf{k}}$ is the type space of data flow $F_{k}$. Then flow $F_{k}$ 's strategy $s_{k}$ is a mapping from $\mathbf{T}_{\mathbf{k}}$ to the strategy space $S$, i.e., $s_{k}^{*}: \mathbf{T}_{\mathbf{k}} \mapsto S$. Besides, the flow rate would be $r_{k}(t)$, the packet size would be $\mu_{k}(t)$, and the energy costs in channel $j$ over link $e$ would be $\varphi_{e, j}^{k}(t)$ if data flow $F_{k}$ is of type $t$. Similarly, we define $\omega_{k}(t)=\frac{\mu_{k}(t)}{r_{k}(t)}$ and denote $T=\left\{t_{1}, \cdots, t_{M}\right\}$ the type profile, where $t_{k}$ is the type of $F_{k}$. Secondly, each player only knows the type distribution $\mathbf{p}$ of other data flows over the type space $\mathbf{T}$, where $\mathbf{p}=\left(p\left(t_{1}, t_{2}, \cdots, t_{M}\right)\right)_{T \in \mathbf{T}}$. Note that the Probability Density Function will be used when the type distribution is continuous. We assume the type distribution of each data flow is independent:

$$
p\left(\hat{t}_{1}, \hat{t}_{2}, \cdots, \hat{t}_{M}\right)=\prod_{k \in \mathcal{M}} p_{k}\left(\hat{t}_{k}\right)
$$

where $p_{k}\left(\hat{t}_{k}\right)$ is the probability that data flow $F_{k}$ is of type $\hat{t}_{k}$, shown by

$$
p_{k}\left(\hat{t}_{k}\right)=\sum_{T \in \mathbf{T}: t_{k}=\hat{t}_{k}} p\left(t_{1}, t_{2}, \cdots, t_{M}\right)
$$

Then we define the concept of equilibria in incompleteinformation games, referred as the Bayesian Nash Equilibrium (BNE).

Definition 5 (Bayesian Nash Equilibrium, BNE): A strategy profile $s^{*}=\left(s_{1}^{*}, s_{2}^{*}, \cdots, s_{M}^{*}\right)$ is a Bayesian Nash Equilibrium if for any data flow $F_{k}(\forall k \in \mathcal{M})$ and its any type $t \in \mathbf{T}_{\mathbf{k}}, s_{k}^{*}(t)$ satisfies:

$$
s_{k}^{*}(t)=\arg \min _{s_{k}(t) \subseteq S} \mathbb{E}\left\{T C_{k}\left(s_{k}(t) ; s_{-k}^{*}\left(t_{-k}\right)\right) \mid t_{k}=t\right\},
$$

where $\mathbb{E}\left\{T C_{k}\left(s_{k}(t) ; s_{-k}^{*}\left(t_{-k}\right)\right) \mid t_{k}=t\right\}$ is $F_{k}$ 's expected costs when it is of type $t$ and adopts strategy $s_{k}(t)$ (note that $t_{-k}$ is a random vector).

Unlike Theorem 2 in the complete-information scenario, we skip the direct proof to the existence of BNE. Instead, we'll first provide an algorithm to compute BNE and then prove its correctness, shown in Algorithm 2 and Theorem 6.

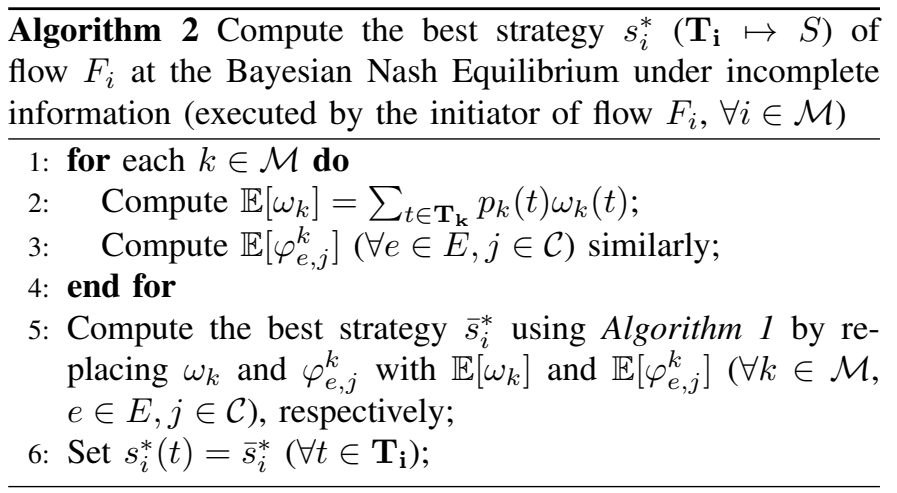

The idea behind Algorithm 2 is simple: each player first computes the expectations of parameters related to other players (as a belief or prediction about them); then each player calls Algorithm 1 by taking in such a belief and derives an equilibrium. Theorem 6 demonstrates that the derived equilibrium is a legitimate BNE.

Theorem 6: Algorithm 2 yields a Bayesian Nash Equilibrium of the Routing-Switching Game with incomplete information.

Proof: Without loss of generality, we assume $\Omega_{R}=$ $\Omega_{S}=1$ here. We consider the contradiction and suppose there exists a data flow $F_{k}$ (of type $t$ ) whose strategy obtained by Algorithm 2 (i.e., $s_{k}^{*}(t)=\bar{s}_{k}^{*}$ ) is not its best response at the Bayesian NE. Then according to the definition of the Bayesian $\mathrm{NE}, F_{k}$ can change its strategy to $\bar{s}_{k}^{\prime}\left(\bar{s}_{k}^{\prime} \neq \bar{s}_{k}^{*}\right)$ so that

$$
\begin{aligned}
\mathbb{E}\left\{T C_{k}\left(\bar{s}_{k}^{\prime} ; s_{-k}^{*}\left(t_{-k}\right)\right) \mid t_{k}\right. & =t\} \\
<\mathbb{E}\left\{T C_{k}\left(\bar{s}_{k}^{*} ; s_{-k}^{*}\left(t_{-k}\right)\right) \mid t_{k}\right. & =t\} .
\end{aligned}
$$

$F_{k}$ 's expected costs when it is of type $t$ under $s_{k}(t)$ are

$$
\begin{aligned}
& \mathbb{E}\left\{T C_{k}\left(s_{k}(t) ; s_{-k}\left(t_{-k}\right)\right) \mid t_{k}=t\right\} \\
= & \sum_{(e, j) \in s_{k}(t)}\left[\mathbb{E}\left\{\lambda_{e, j}^{k}\left(s_{-k}\left(t_{-k}\right)\right)\right\}+\varphi_{e, j}^{k}(t)+\gamma_{e, j}\right] .
\end{aligned}
$$


Note that equation (20) is established on the fact that $\mathbb{E}\left\{\lambda_{e, j}^{k}\left(s_{k}(t) ; s_{-k}\left(t_{-k}\right)\right) \mid t_{k}=t\right\}=\mathbb{E}\left\{\lambda_{e, j}^{k}\left(s_{-k}\left(t_{-k}\right)\right)\right\}$ since $\lambda_{e, j}^{k}(s)$ only depends on others' strategies $s_{-k}\left(t_{-k}\right)$ and the type distribution of each data flow is statistically independent. Taking (20) to (19), we derive

$$
\begin{aligned}
& \sum_{(e, j) \in \bar{s}_{k}^{\prime}}\left[\mathbb{E}\left\{\lambda_{e, j}^{k}\left(s_{-k}^{*}\left(t_{-k}\right)\right)\right\}+\varphi_{e, j}^{k}(t)+\gamma_{e, j}\right] \\
< & \sum_{(e, j) \in \bar{s}_{k}^{*}}\left[\mathbb{E}\left\{\lambda_{e, j}^{k}\left(s_{-k}^{*}\left(t_{-k}\right)\right)\right\}+\varphi_{e, j}^{k}(t)+\gamma_{e, j}\right] .
\end{aligned}
$$

Step 5 in Algorithm 2 corresponds to a new completeinformation game. To avoid the confusion of denotations, we will denote $\bar{s}$ an arbitrary strategy profile in the new complete-information game and the corresponding 0-1 strategy indication is $\bar{X}_{e, j}^{k^{\prime}}\left(\forall k^{\prime} \in \mathcal{M}, e \in E, j \in \mathcal{C}\right)$. By comparison, the corresponding strategy profile in the incompleteinformation game is $s$ which is a mapping from the type space to the strategy space, and the $0-1$ indication is $X_{e, j}^{k^{\prime}}(t)$ $\left(\forall k^{\prime} \in \mathcal{M}, e \in E, j \in \mathcal{C}, t \in \mathbf{T}_{\mathbf{k}^{\prime}}\right)$. By this definition, in the new complete-information game,

$$
T C_{k}(\bar{s})=\sum_{(e, j) \in \bar{s}_{k}}\left[\bar{\lambda}_{e, j}^{k}(\bar{s})+\varphi_{e, j}^{k}(t)+\gamma_{e, j}\right] .
$$

Here,

$$
\begin{gathered}
\bar{\lambda}_{e, j}^{k}(\bar{s})=\sum_{e^{\prime} \in E} \sum_{k^{\prime} \in \mathcal{M}_{k}} \bar{X}_{e^{\prime}, j}^{k^{\prime}} \bar{\omega}_{k^{\prime}} \theta_{e, e^{\prime}}, \\
\bar{\omega}_{k^{\prime}}=\mathbb{E}\left[\omega_{k^{\prime}}\right]=\sum_{t^{\prime} \in \mathbf{T}_{\mathbf{k}^{\prime}}} p_{k^{\prime}}\left(t^{\prime}\right) \omega_{k^{\prime}}\left(t^{\prime}\right) .
\end{gathered}
$$

The above equation is actually derived from step 2 of Algorithm 2. By equation (18), we know that

$$
\begin{aligned}
\bar{\omega}_{k^{\prime}} & =\sum_{t^{\prime} \in \mathbf{T}_{\mathbf{k}^{\prime}}} p_{k^{\prime}}\left(t^{\prime}\right) \omega_{k^{\prime}}\left(t^{\prime}\right) \\
& =\sum_{t^{\prime} \in \mathbf{T}_{\mathbf{k}^{\prime}}} \sum_{T \in \mathbf{T}: t_{k^{\prime}}=t^{\prime}} p\left(t_{1}, \cdots, t_{M}\right) \omega_{k^{\prime}}\left(t^{\prime}\right) \\
& =\sum_{T \in \mathbf{T}} p\left(t_{1}, \cdots, t_{M}\right) \omega_{k^{\prime}}\left(t_{k^{\prime}}\right) .
\end{aligned}
$$

Then $\bar{\lambda}_{e, j}^{k}(\bar{s})$ can be rewritten as

$$
\begin{aligned}
\bar{\lambda}_{e, j}^{k}(\bar{s}) & =\sum_{e^{\prime} \in E} \sum_{k^{\prime} \in \mathcal{M}_{k}} \bar{X}_{e^{\prime}, j}^{k^{\prime}} \theta_{e, e^{\prime}} \sum_{T \in \mathbf{T}} p\left(t_{1}, \cdots, t_{M}\right) \omega_{k^{\prime}}\left(t_{k^{\prime}}\right) \\
& =\sum_{T \in \mathbf{T}} p\left(t_{1}, \cdots, t_{M}\right) \sum_{e^{\prime} \in E} \sum_{k^{\prime} \in \mathcal{M}_{k}} X_{e^{\prime}, j}^{k^{\prime}}\left(t_{k^{\prime}}\right) \theta_{e, e^{\prime}} \omega_{k^{\prime}}\left(t_{k^{\prime}}\right) \\
& =\mathbb{E}\left\{\lambda_{e, j}^{k}\left(s_{-k}\left(t_{-k}\right)\right)\right\},
\end{aligned}
$$

where the second equality holds because any other flows follow Algorithm 2 and step 6 shows that $\bar{s}_{k^{\prime}}=s_{k^{\prime}}\left(t_{k^{\prime}}\right)$ (i.e., $\left.\bar{X}_{e^{\prime}, j}^{k^{\prime}}=X_{e^{\prime}, j}^{k^{\prime}}\left(t_{k^{\prime}}\right)\right)$ for every $k^{\prime} \in \mathcal{M}_{k}$ and $t_{k^{\prime}} \in \mathbf{T}_{\mathbf{k}^{\prime}}$ in the new complete-information game. This further implies that

$$
T C_{k}(\bar{s})=\sum_{(e, j) \in \bar{s}_{k}}\left[\mathbb{E}\left\{\lambda_{e, j}^{k}\left(s_{-k}\left(t_{-k}\right)\right)\right\}+\varphi_{e, j}^{k}(t)+\gamma_{e, j}\right]
$$

Taking the above equation to (21) and noticing that $s_{-k}^{*}\left(t_{-k}\right)=\bar{s}_{-k}^{*}$ for every $t_{-k} \in \mathbf{T}_{-\mathbf{k}}$, we can conclude that in the new complete-information game formed in step 5 of Algorithm 2:

$$
T C_{k}\left(\bar{s}_{k}^{\prime} ; \bar{s}_{-k}^{*}\right)<T C_{k}\left(\bar{s}_{k}^{*} ; \bar{s}_{-k}^{*}\right)
$$

which means that $\bar{s}^{*}$ is not the NE in the corresponding complete-information game, contradicting to the correctness of Algorithm 1. Hence Theorem 6 has been proved.

It should be mentioned that when the Bayesian $\epsilon$-Nash Equilibrium is calculated, similar modification (see section III-D) should be made to Algorithm 1. Besides, since statistical information is used in the Fictitious Play Process, each player cannot precisely simulate others' actions, which implies that there are certain performance gaps between the BNE and the NE (see section VII for numerical demonstrations).

\section{PRice of Stability}

In this section, we will compare the performance of the proposed game with the socially optimal results obtained in centralized schemes. As for the complete-information game, Price of Stability $(\mathrm{PoS})$ in terms of social costs will be analyzed. In terms of the incomplete-information scenario, expected social costs as well as Bayesian Price of Stability $(\mathrm{BPoS})$ will be discussed.

\section{A. Complete-Information Game}

In the route-switching game with complete information, the metric of our interests is social costs, defined as the following.

Definition 6 (Social Costs): Social costs are the sum of all players' overall costs, i.e.,

$$
\operatorname{SoC}(s)=\sum_{k \in \mathcal{M}} T C_{k}(s) .
$$

Then we introduce the definition of Price of Stability in the complete-information game.

Definition 7 (Price of Stability): Price of Stability is the ratio of social costs between the best NE and the optimality in centralized schemes, i.e.,

$$
P o S=\frac{S o C\left(s^{*}\right)}{\min _{s} S o C(s)},
$$

where $s^{*}$ is the best NE point of the proposed game.

The following theorem shows that PoS of the proposed game has an upper bound.

Theorem 7: The upper bound of Price of Stability in the proposed game is $\rho$, where $\rho=2 \max _{k, l \in \mathcal{M}} \frac{\omega_{k}}{\omega_{l}}$.

Proof: With loss of generality, we set $\Omega_{R}=\Omega_{S}=1$. Let $s^{*}$ be the best Nash Equilibrium of the game and $s^{\prime}$ be the NE that globally minimizes the potential function. Besides, let $q$ be the strategy profile which can minimize the social costs. At the same time, we define $Z_{1}:=\max _{k} \omega_{k}$ and $Z_{2}:=\min _{k} \omega_{k}$. Thus $\rho=\frac{2 Z_{1}}{Z_{2}}$. We can rewrite the potential function as

$$
\begin{aligned}
\Phi(s) & =2 \sum_{k \in \mathcal{M}} \omega_{k}\left[D C_{k}(s)+E C_{k}(s)+S C_{k}(s)\right]-\sum_{k \in \mathcal{M}} \omega_{k} D C_{k}(s) \\
& \leq 2 Z_{1} S o C(s)-\sum_{k \in \mathcal{M}} \omega_{k} D C_{k}(s)
\end{aligned}
$$


Similarly, we have

$$
\Phi(s) \geq Z_{2} S o C(s)+\sum_{k \in \mathcal{M}} \omega_{k}\left[E C_{k}(s)+S C_{k}(s)\right] .
$$

Since $\Phi\left(s^{\prime}\right)$ reaches the global minimum, we have

$$
\begin{aligned}
& Z_{2} S o C\left(s^{\prime}\right)+\sum_{k \in \mathcal{M}} \omega_{k}\left[E C_{k}\left(s^{\prime}\right)+S C_{k}\left(s^{\prime}\right)\right] \leq \Phi\left(s^{\prime}\right) \\
& \leq \Phi(q) \leq 2 Z_{1} S o C(q)-\sum_{k \in \mathcal{M}} \omega_{k} D C_{k}(q) .
\end{aligned}
$$

For the simplicity of denotations, we define

$$
\alpha:=\frac{\sum_{k} \omega_{k}\left[D C_{k}(q)+E C_{k}\left(s^{\prime}\right)+S C_{k}\left(s^{\prime}\right)\right]}{\operatorname{SoC}(q)}
$$

Then we can derive that

$$
Z_{2} \operatorname{SoC}\left(s^{\prime}\right) \leq \operatorname{SoC}(q)\left(2 Z_{1}-\alpha\right)
$$

Since $s^{*}$ is the best Nash Equilibrium, then $\operatorname{SoC}\left(s^{*}\right) \leq$ $\operatorname{SoC}\left(s^{\prime}\right)$. Thus, we finally have

$$
P o S=\frac{\operatorname{SoC}\left(s^{*}\right)}{\operatorname{SoC}(q)} \leq \frac{\operatorname{SoC}\left(s^{\prime}\right)}{\operatorname{SoC}(q)} \leq \frac{2 Z_{1}}{Z_{2}}-\frac{\alpha}{Z_{2}} \leq \rho
$$

Theorem 7 implies that social costs under the best NE won't exceed $\rho$ times of the minimum social costs. Here, $\rho$ characterizes the heterogeneity of traffic demands from incoming data flows. In practical communications systems, such heterogeneity is not significant considering transmission efficiency [31]. In a special case when flows are homogeneous ( $\omega_{k}$ is identical, $\forall k \in \mathcal{M}$ ), the best NE yields less than twice of the minimum social costs $(\rho=2)$. Besides, it should be mentioned that $\rho$ is a relatively loose bound, which means that the real PoS could be much less than $\rho$. The above two remarks of $\rho$ imply that the obtained NE is actually close to the optimality in practice ( $\mathrm{PoS}$ is usually below 1.5 in our simulation, see section VII).

\section{B. Incomplete-Information Game}

As for the incomplete-information scenario, the corresponding concept is referred as the Bayesian Price of Stability $(\mathrm{BPoS})$, which is defined as the following.

Definition 8 (Bayesian Price of Stability): Bayesian price of Stability is the ratio of expected social costs between the best Bayesian NE and the optimal results obtained by centralized schemes, i.e.,

$$
B P o S=\frac{\mathbb{E}\left\{S o C\left(s^{*}\right)\right\}}{\min _{s} \mathbb{E}\{S o C(s)\}},
$$

where the expectations are over the entire type space T. A deterministic upper bound of Bayesian Price of Stability is given in Theorem 8 .

Theorem 8: The upper bound of Bayesian Price of Stability in the proposed game is $\varrho$, where $\varrho=2 \max _{k, l \in \mathcal{M}} \frac{\mathbb{E}\left[\omega_{k}\right]}{\mathbb{E}\left[\omega_{l}\right]}$, where the expectations are over the type space of flows $F_{k}$ and $F_{l}$, respectively.

The proof to Theorem 8 is similar to that of Theorem 7 except some lengthy probabilistic calculations. Due to the limited space, we omit the proof here. Theorem 8 implies that $\mathrm{BPoS}$ in the proposed game is also related to the (statistical) heterogeneity of incoming data flows. Besides, similar to the discussion of PoS, the real BPoS is not significant in practical systems.

\section{DISCUSSION}

\section{A. Tradeoff between Routing Costs and Switching Costs}

In this subsection, we discuss the tradeoff between routing and switching costs. Due to the limited space, a brief heuristic demonstration is provided instead of a formal mathematical analysis.

Suppose the flow of our interests is $F_{k}$. For simplicity, we assume each link only sustains one channel such that the maximum number of channel switching is $|E|$. Denote $Z$ the allowable number of channel switching in $F_{k}$ 's route ( $Z=0,1, \cdots,|E|$ ). Then $Z \gamma$ roughly reflects the total switching costs incurred to flow $F_{k}$. Besides, suppose the set of channels reclaimed by PUs is $\Gamma$ and the set of invalid links due to such spectrum mobility is $\mathcal{E}=\left\{e \in E \mid \exists j \in \Gamma, A_{e, j}=1\right\}$.

When $Z=0$, i.e., switching costs achieve the minimum (zero), the initiator of $F_{k}$ has to select a new spatial route in the new graph $G_{0}=(V, E \backslash \mathcal{E})$. We denote the optimal routing costs in $G_{0}$ as $R C_{k}^{(0)}$.

When $Z=1$, switching costs increase, but the initiator of $F_{k}$ has the flexibility of switching one channel, where the resulting new graph $G_{1}$ has two possibilities. In the first case, the initiator of $F_{k}$ switches the channel over a certain edge $e_{1} \in \mathcal{E}$, and thus link $e_{1}$ is available again, which implies $G_{1}=\left(V,(E \backslash \mathcal{E}) \cup\left\{e_{1}\right\}\right)$ and $G_{0} \subset G_{1}$. Hence, denoting the optimal routing costs in $G_{1}$ as $R C_{k}^{(1)}$, we obtain $R C_{k}^{(0)} \geq R C_{k}^{(1)}$, where "=" only happens when resources (e.g., available channels and links) are abundant. In the second case, the initiator of $F_{k}$ switches the channel over a certain edge $e_{1} \in E \backslash \mathcal{E}$. This happens when this channel is perceived to be overly congested over $e_{1}$ and routing costs can be significantly reduced if we switch the assigned channel of $e_{1}$ to a less congested one, where $R C_{k}^{(0)}>R C_{k}^{(1)}$ also holds. Therefore, regardless of the switching choice, we always have $R C_{k}^{(0)}>R C_{k}^{(1)}$ when network resources are limited.

Similar argument holds when we raise $Z$ until $Z=|E|$. Then we can conclude that the increase of switching costs can reduce routing costs. Conversely, we can similarly show that the increase of routing costs also contributes to the reduction of switching costs. Therefore, routing costs and switching costs cannot be simultaneously minimized and tradeoffs exists between them. In section VII, we will further provide numerical results to illustrate such tradeoffs adjusted by parameters $\Omega_{R}$ and $\Omega_{S}$.

\section{B. Implementation of the Game}

We briefly discuss the implementation of the proposed game. Before flow transmission, nodes (i.e., SUs) will first perform spectrum sensing to obtain the states of channels ${ }^{12}$.

\footnotetext{
${ }^{12}$ To reduce sensing overheads, efficient sensing schemes like cooperative sensing [13] and compressive sensing [14] can be adopted here.
} 
If the available channels cannot sustain existing routes, nodes where the routes break will broadcast route-switching messages to flow initiators through the flooding scheme. Each route-switching message contains the indices of affected flows as well as the indices of channels whose state changes so that each initiator can obtain $\Gamma$ (i.e., the set of unavailable channels). Then initiators will distributively play the RouteSwitching Game to re-select their two-dimensional routes and inform the intermediate nodes of the new decisions. Here, initiators can include the new route table in the header of packets like in the Dynamic Source Routing (DSR) protocol. Finally, new routes are built and flow transmission resumes.

Note that when PUs suddenly reclaim their licensed channels, there might be some packets in the middle of delivery whose old route breaks while the new route is still unknown to them. In this case, there are several possible solutions. For example, the simplest way is that intermediate nodes drop the undelivered packets and the source nodes retransmit the packets whose ACK is not received. Alternatively, intermediate nodes can temporarily resume the transmission of undelivered packets using backup channels (e.g., [16]), until all undelivered packets are cleared. Since we are considering constant flows and the frequency of PUs' channel reclamation is relatively low (e.g., in TV whitespace, the time interval between two consecutive PUs' activities is usually on order of minutes or hours [36]), such a temporary state won't cause significant degradations to network performances.

Besides, our scheme also requires that each secondary node has the complete topology information of the network, which is challenging in practice. Fortunately, the draft of IEEE 802.22 [37] points out that PUs can periodically send beacon frames to SUs, which provides an option to contain the locations of interfering SUs. Moreover, although the spectrum occupancy in CRNs is time-varying, the underlying topology (e.g., the locations of SUs and their interfering relationship) can be relatively static (we do not consider node mobility in this paper). In this case, even without aforementioned beacon frames, it is also possible to statically maintain the topology information at each node (e.g., pre-store the topology information in the database of each secondary node [9] [16]).

\section{Simulation}

\section{A. Simulation Settings}

In this paper, we use MATLAB as the simulation tool. As for the network topology, we adopt the classical B-A algorithm to generate a (random) scale-free network. We also randomly assign a distance for each pair of nodes in the generated network following the uniform distribution, with the distribution interval $[1,20] \mathrm{m}$ for the first-hop, $[20,40] \mathrm{m}$ for the second-hop, etc. The interference range of each node is $60 \mathrm{~m}$. The number of radios equipped by each SU is a random integer following the uniform distribution between $[1,6]$. Flow rates and packet sizes are uniformly distributed in $[0.8,1.2] \mathrm{Mbps}$ and $[600,800]$ Bytes, respectively. As for power consumption, we use the simple form mentioned in section II-E. Besides, the old channel assignment (i.e., $A_{e, j}, \forall e \in E, j \in \mathcal{C}$ ) as well as the current state of each licensed channel is 0 or 1 with

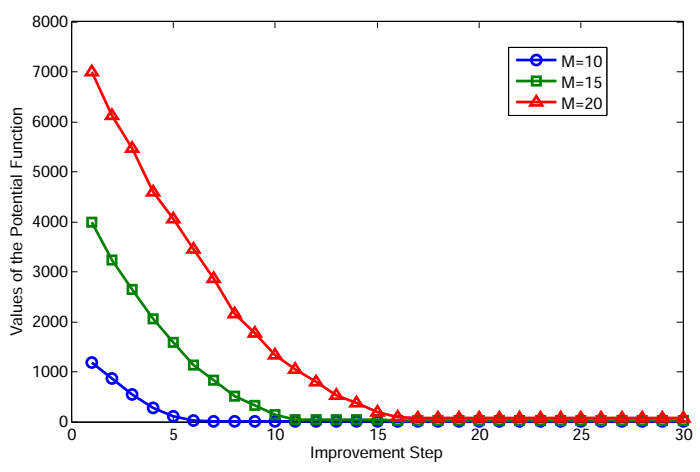

Fig. 3. Finite Improvement Property of the proposed game.

an equal probability 0.5 . In the following, the numbers of total channels and nodes in the network (i.e., $C$ and $|V|$ ) are fixed to be 5 and 10, respectively. The marginal costs $P_{d}, P_{e}$ and the tradeoff parameters $\Omega_{R}, \Omega_{S}$ are all set to be 1 unless particularly stated.

\section{B. Simulation Results}

We first simulate the Finite Improvement Property of the Route-Switching Game, shown in Figure 3. Initially, each player randomly picks a two-dimensional route, which incurs a large potential value. After each improvement step, the potential is gradually reduced and finally reaches the minimum (a very small value but not zero, which might not be obvious in the figure due to the numerical scale), where a pure NE is reached. It also shows that games with more players require more improvement steps but the minimum can still be quickly reached (less than 30 iterations for 20 flows).

We then focus on the performance of the $\epsilon-\mathrm{NE}$, which sacrifices some precision in return for time efficiency. Figure 4 shows the average number of improvement steps under different $\epsilon$ (note that $\epsilon=0$ corresponds with the exact Nash Equilibrium). Dotted lines show the confidence interval in 50 experiments. We can apparently observe that the number of improvement steps reduces significantly with the increase of $\epsilon$. Besides, we also compare the precision of equilibria obtained with different $\epsilon$ in Figure 5, which reveals that the potential is raised almost linearly with the increase of $\epsilon$, i.e., the precision of the obtained equilibrium drops. Therefore, in practice, careful design of $\epsilon$ is required to achieve the balance between time efficiency and precision.

In Figure 6, we illustrate the comparison between the socially optimal results (obtained by exhaustive search) and the Nash Equilibria of the proposed game (obtained by Algorithm 1) in terms of social costs. We can observe that the Nash equilibria yield more social costs than the optimal results, which is due to the lack of cooperation among different flows. However, their performance gap is not significant, and it turns out that the average $\mathrm{PoS}$ is below 1.6. By comparison, Theorem 7 indicates that the theoretical bound of $\operatorname{PoS}$ is 2 under our simulation settings. Hence, the practical performance of the $\mathrm{NE}$ is usually better than the theoretical bound.

Next, social costs incurred under complete and incomplete information are compared, as is shown in Figure 7. Note 


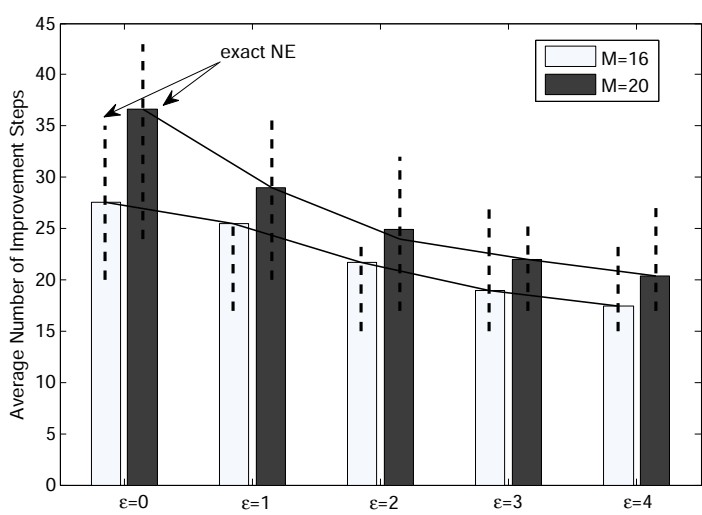

Fig. 4. Average number of improvement steps used for finding the $\epsilon$-NE. Dotted lines show the confidence interval in 50 experiments.

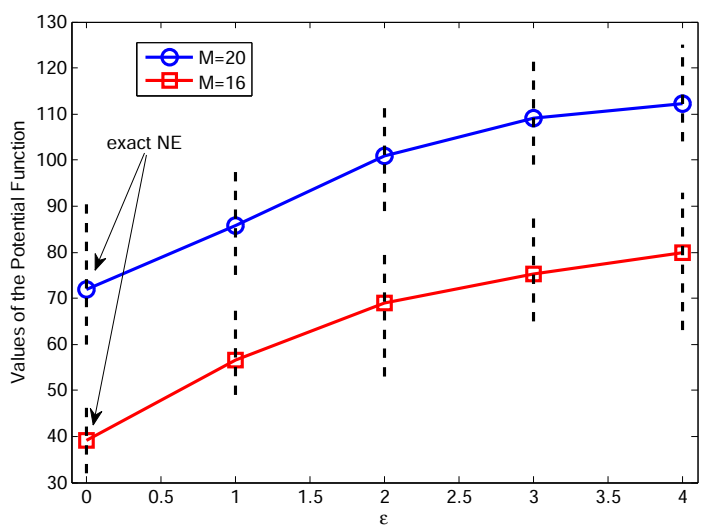

Fig. 5. Potential values of $\epsilon$-Nash Equilibria under different $\epsilon$.

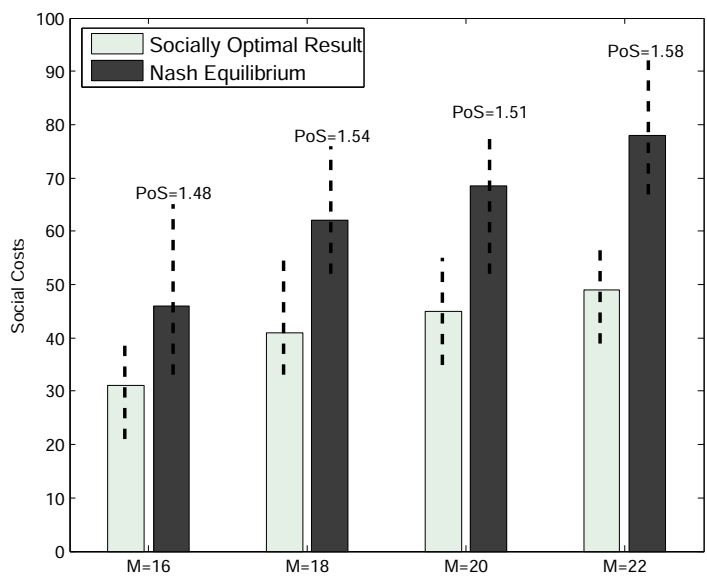

Fig. 6. Comparison between the socially optimal results and the Nash Equilibria in terms of social costs.

that under complete information, parameters of all flows (i.e., their data rates and packet sizes) are publicly known. By comparison, under incomplete information, these generated parameters are no longer accessible to all players (expect their own parameters), and each player only knows that the data rate and the packet size of other flows are uniformly distributed within $[0.8,1.2] \mathrm{Mbps}$ and $[600,800]$ Bytes, respectively. We can observe that social costs obtained in the complete-information scenario are fewer than those in the incomplete-information

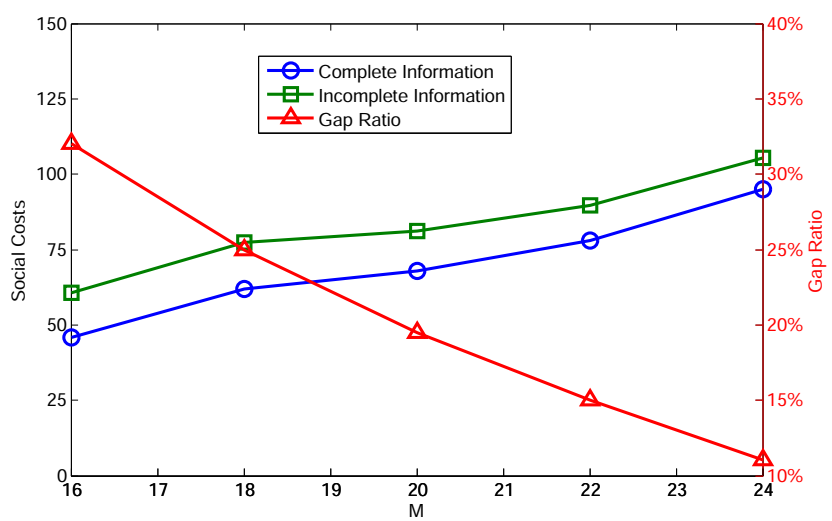

Fig. 7. Comparison of social costs between complete- and incompleteinformation games. Their performance gap (measured in the percentile form) is also demonstrated.

game, which demonstrates the advantage of full knowledge. However, as is illustrated by the red curve (marked by triangles) in Figure 7, the performance gap (measured in the percentile form and in terms of social costs) between the two scenarios becomes less and less significant with more and more flows into the network. That is, the advantage brought by complete information is gradually obscure since players' real type distribution is closer and closer to the probability distribution when more and more players participate in the game.

Finally, we numerically investigate the tradeoffs between routing costs and switching costs, shown in Figure $8(M=$ 20). Here, we achieve different levels of tradeoffs by adapting the values of parameters $\Omega_{R}$ and $\Omega_{S}$. Specifically, when $\Omega_{R}=0$ and $\Omega_{S}=1$, switching costs are minimized while the corresponding routing costs achieve the peak value. When $\Omega_{S}$ is still 1 but $\Omega_{R}$ is raised to 0.5 , we can observe that switching costs increase in return for the significant reduction in routing costs. Further, when the ratio $\Omega_{R} / \Omega_{S}$ continues to grow, routing costs are gradually reduced at the expense of switching costs. Finally, when $\Omega_{R}=1$ and $\Omega_{S}=0$, routing costs are minimized but switching costs reach the highest level. Therefore, as is proved in section VI-A, routing and switching costs cannot be simultaneously minimized, and we can reduce one type of costs by properly increasing the other type of costs, i.e., tradeoffs exist between them.

\section{RELATED WORKS}

As for two-dimensional routing, there has been some literature on the similar problem in conventional wireless networks. A joint channel assignment and routing protocol was investigated by Chiu et al. [2] for the IEEE 802.11-based mobile ad hoc networks. A novel routing metric was introduce by $\mathrm{Wu}$ et al. [3] to design distributed channel assignment and routing in multi-hop wireless networks. Kodialam et al. [5] and Alicherry et al. [6] jointly considered channel assignment and multi-flow scheduling in the mesh networks. Unfortunately, most of these existing works are neither robust enough to handle spectrum mobility in CRNs nor able to weigh the benefits and costs of channel switching. 


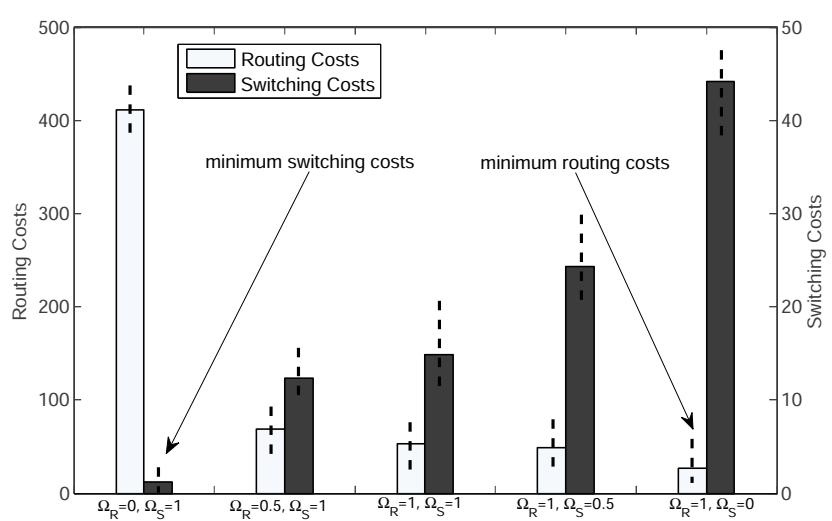

Fig. 8. Tradeoffs between routing costs and switching costs. Note that routing and switching costs cannot be simultaneously minimized. One type of costs can be reduced by raising the other type of costs.

In the context of CRNs, spectrum dynamics have been heatedly studied recently. For example, Southwell et al. [9] proposed spectrum mobility games in CRNs in order to derive a channel switching plan which minimizes the congestion level, and Liang et al. [8] applied game-theoretical approaches to spectrum selection problems in face of channel dynamics. A robust channel assignment scheme in the multi-hop CRN was provided by Zhao et al. [16] to handle PUs' channel reclaiming behaviors. Liu et al. [35] considered both the benefits and prices of channel switching, where an optimal channel access scheme was developed to improve transmission opportunities and mitigate channel congestion. In the spatial domain, Caleffi et al. [12] considered the diversity effects of spatial routes and proposed the criterion for an optimal routing metric in CRNs, and a connectivity-based routing scheme for the cognitive ad hoc networks was introduced in the work of Abbagnale et al. [15]. However, these schemes only considered either the frequency or the spatial domain. Ding et al. [34] proposed a two-dimensional algorithm in CRNs by jointly considering routing, dynamic spectrum allocation, scheduling and power control scheme, which distributively maximizes the throughput of the network while guaranteeing bounded bit error rates. Unfortunately, the influence of channel switching costs were not discussed in this paper. As far as we know, there's still no major work investigating spectrum-mobility-incurred routeswitching problems in both spatial and frequency domains for CRNs.

\section{CONCLUSION}

In this paper, we investigate the spectrum-mobility-incurred route-switching problem in multi-hop CRNs, where a joint scheme of channel reassignment and rerouting is explored. We formulate the proposed problem as the Route-Switching Game and prove that this game has a potential function. Then an iterative algorithm for finding the NE and a polynomial-time algorithm for computing the $\epsilon-\mathrm{NE}$ are provided in the paper. The proposed game is further extended to the incompleteinformation scenario and an algorithm for calculating the Bayesian NE is provided. Finally, we show that price of stability of the proposed game has a deterministic upper bound. Simulation results validate our theoretical analysis and demonstrate the tradeoffs between routing and switching costs.

\section{ACKNOWLEDGMENT}

This work is supported by NSF China (No. 61325012, 61271219); China Ministry of Education Fok Ying Tung Fund (No. 122002); China Ministry of Education New Century Excellent Talent (No. NCET-10-0580) China Ministry of Education Doctor Program (No. 20130073110025) Shanghai Basic Research Key Project (No.11JC1405100) Shanghai International Cooperation Project: (No. 13510711300)

\section{REFERENCES}

[1] J. Mitola, Cognitive Radio. John Wiley and Sons, 2006.

[2] H. S. Chiu, K. L. Yeung and K. S. Lui, "J-CAR: An efficient joint channel assignment and routing protocol for IEEE 802.11-based multichannel multi-interface mobile Ad Hoc networks," in IEEE Transactions on Wireless Communications, vol.8, no.4, pp.1706-1715, 2009.

[3] H. Wu, F. Yang, K. Tan, J. Chen, Q. Zhang and Z. Zhang, "Distributed Channel Assignment and Routing in Multiradio Multichannel Multihop Wireless Networks," in IEEE Journal on Selected Areas in Communications, vol.24, no.11, pp.1972-1983, 2006.

[4] Q. Liang, X. Wang, X. Tian and Q. Zhang, "Route-Switching Games in Cognitive Radio Networks," in Proceedings of ACM MobiHoc, 2013.

[5] M. Kodialam and T. Nandagopal, "Characterizing the Capacity Region in Multi-Radio Multi-Channel Wireless Mesh Networks," in Proceedings of ACM MobiCom, 2005.

[6] M. Alicherry, R. Bhatia and L. Erran, "Joint Channel Assignment and Routing for Throughput Optimization in Multiradio Wireless Mesh Networks," in IEEE Journal on Selected Areas in Communications, vol.24, no.11, pp.1960-1971, 2006.

[7] P. Gupta and P. R. Kumar, "The Capacity of Wireless Networks," in IEEE Transactions on Information Theory, vol.46, no.2, pp.388-404, 2000.

[8] Q. Liang, X. Wang and Z. Feng, "Singleton Spectrum Mobility Games With Incomplete Information," in Proceedings of IEEE Globecom, 2012.

[9] R. Southwell, J. Huang and X. Liu, "Spectrum Mobility Games," in Proceedings of IEEE INFOCOM, 2012.

[10] S. Yarkan and H. Arslan, "Binary time series approach to spectrum prediction for cognitive radio," in Proceedings of IEEE Vehicular Technology Conference, 2007.

[11] A. Gopinathan and Z. Li, "Strategyproof auctions for balancing social welfare and fairness in secondary spectrum markets," in Proceedings of IEEE INFOCOM, 2011.

[12] M. Caleffi, I. F. Akyildiz and L. Paura, "OPERA: Optimal Routing Metric for Cognitive Radio Ad Hoc Networks," in IEEE Transactions on Wireless Communications, vol.11, no.8, August 2012.

[13] T. Yucek and H. Arslan, "A survey of spectrum sensing algorithms for cognitive radio applications," in IEEE Communications Surveys Tutorials, vol.11, no.1, pp.116-130, 2009.

[14] F. Zeng, C. Li and Z. Tian, "Distributed Compressive Spectrum Sensing in Cooperative Multihop Cognitive Networks," in IEEE Journal of Selected Topics in Signal Processing, vol.5, no.1, pp.37-48, 2011.

[15] A. Abbagnale and F. Cuomo, "Gymkhana: A Connectivity Based Routing Scheme for Cognitive Radio Ad Hoc Networks," in Proceedings of IEEE INFOCOM, 2010.

[16] J. Zhao and G. Cao, "Robust Topology Control in Multi-hop Cognitive Radio Networks," in Proceedings of IEEE INFOCOM, 2012.

[17] D. Choi and D. Park, "Effective self interference cancellation in full duplex relay systems," in Electronics Letter, vol.48, no.2, January 2012.

[18] B. Chun, E. R. Jeong, J. Joung, Y. Oh and Y. H. Lee, "Pre-Nulling for Self-Interference Suppression in Full-Duplex Relays," in APSIPA Annual Summit and Conference, 2009.

[19] X. Chen and J. Huang, "Spatial Spectrum Access Game: Nash Equilibria and Distributed Learning," in Proceedings of ACM MobiHoc, 2012.

[20] N. Nie and C. Comniciu, "Adaptive channel allocation spectrum etiquette for cognitive radio networks," IEEE DySPAN, 2005.

[21] Y. Song, H. Y. Wong and K. W. Lee, "Optimal Gateway Selection in Multi-domain Wireless Networks: A Potential Game Perspective," in Proceedings of ACM MobiCom, 2011.

[22] F. Wu, N. Singh, N. Vaidya and G. Chen, "On Adaptive-Width Channel Allocation in Non-Cooperative, Multi-Radio Wireless Networks," in Proceedings of IEEE INFOCOM, 2011. 
[23] F. Wu, S. Zhong and C. Qiao, "Globally optimal channel assignment for non-cooperative wireless networks," in Proceedings of IEEE INFOCOM. 2008.

[24] N. Abramso, "The Aloha System: another alternative for computer communications," in Proceedings of ACM AFIPS, 1970.

[25] W. Stallings, Wireless Communications \& Networks, 2nd edition. Prentice Hall, 2005.

[26] M. R. Chari, F. Ling, A. Mantravadi, R. Krishnamoorthi, R. Vijayan, G. K. Walker and R. Chandhok, "FLO Physical Layer: An Overview," IEEE Trans. Broadcasting, vol.53, no.1, pp.145-159, 2007.

[27] W. Wang, S. Eidenbez, Y. Wang and X. Y. Li, "Ours-optimal unicast routing systems in non-cooperative wireless networks," in Proceedings of ACM MobiCom, 2006.

[28] Q. Liang, S. Han, F. Yang, G. Sun and X. Wang, "A DistributedCentralized Scheme for Short- and Long-Term Spectrum Sharing with a Random Leader in Cognitive Radio Networks," in IEEE Journal on Sel. Areas in Comm., vol. 30, no. 11, pp. 2274-2284, 2012.

[29] H. Li, Y. Cheng, C. Zhou, and W. Zhuang, "Minimizing End-toEnd Delay: A Novel Routing Metric for Multi-Radio Wireless Mesh Networks", in Proc. of IEEE INFOCOM, 2009.

[30] C. Sarr and I. Guerin, "Estimating Average End-to-End Delays in IEEE 802.11 Multihop Wireless Networks," technical report, CITI Insa Lyon/INRIA Grenoble Rhone-Alpes-ARES, 2007.

[31] T. Rappaport, Wireless communications: Principles and Practice. Prentice Hall, 2002.

[32] G. Christodoulou and E. Koutsoupias, "The price of anarchy of finite congestion games," in Proceedings of ACM STOC, 2005.

[33] D. Monderer and L. S. Shapley, "Potential Games", in Games and Economic Behavior, vol.14, pp.124-143, 1996.

[34] L. Ding, T. Melodia, S. Batalama, J. Matyjas and M. Medley, "Crosslayer Routing and Dynamic Spectrum Allocation in Cognitive Radio Ad Hoc Networks," in IEEE Transactions on Vehicular Technology, vol.59, no.4, pp.1969-1979, 2010.

[35] Y. Liu and M. Liu, "To Stay Or To Switch: Multiuser Dynamic Channel Access," in Proc. of IEEE Infocom, 2013.

[36] C. Cordeiro, K. Challapali, D. Birru and N. S. Shankar, "IEEE 802.22: the first worldwide wireless standard based on cognitive radios," In Proc. of IEEE DYSPAN, 2005.

[37] IEEE 802.22 Working Group, "Part 22: Cognitive Wireless RAN Medium Access Control (MAC) and Physical Layer (PHY) Specifications: Policies and Procedures for Operation in the TV Bands", IEEE, 2011.

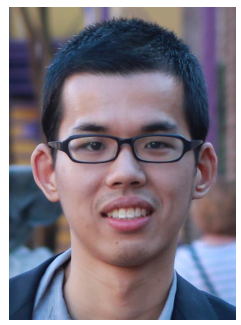

Qingkai Liang received the B.E. degree (with the highest honor) from the Department of Electronic Engineering, Shanghai Jiao Tong University, Shanghai, China, in 2013. Currently, he is a graduate student at Massachusetts Institute of Technology. His research interests include the theory of network control and optimization with their applications in practical networked systems.

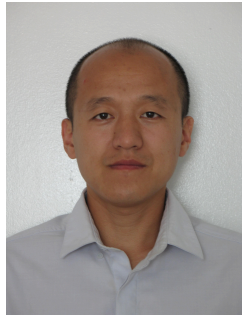

Xinbing Wang received the B.S. degree (with honors.) from the Department of Automation, Shanghai Jiaotong University, Shanghai, China, in 1998, and the M.S. degree from the Department of Computer Science and Technology, Tsinghua University, Beijing, China, in 2001. He received the Ph.D. degree, major in the Department of electrical and Computer Engineering, minor in the Department of Mathematics, North Carolina State University, Raleigh, in 2006. Currently, he is a professor in the Department of Electronic Engineering, Shanghai Jiaotong University, Shanghai, China. Dr. Wang has been an associate editor for IEEE/ACM Transactions on Networking and IEEE Transactions on Mobile Computing, and the member of the Technical Program Committees of several conferences including ACM MobiCom 2012,2014, ACM MobiHoc 20122014, IEEE INFOCOM 2009-2014.

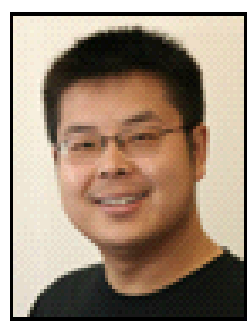

Xiaohua Tian received his B.E. and M.E. degrees in communication engineering from Northwestern Polytechnical University, Xian, China, in 2003 and 2006, respectively. He received the Ph.D. degree in the Department of Electrical and Computer Engineering (ECE), Illinois Institute of Technology (IIT), Chicago, in Dec. 2010. He is currently an Assistant Professor in Department of Electronic Engineering of Shanghai Jiao Tong University, China. He won the Highest Standards of Academic Achievement 2011 of IIT and Fieldhouse Research Fellowship 2009, which is awarded to only one student over IIT each year. His research interests include application-oriented networking, Internet of Things and wireless networks. He serves as the guest editor of International Journal of Sensor Networks, publicity co-chair of WASA 2012, local management chair of IEEE/CIC ICCC 2014. He also serves as the symposium chair of WASA 2014, the Technical Program Committee member for IEEE INFOCOM 2014, IEEE GLOBECOM 2011-2014, IEEE ICC 2012-2014, and WASA 2011.

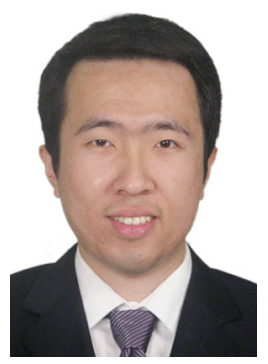

Fan Wu is an associate professor in the Department of Computer Science and Engineering at Shanghai Jiao Tong University, P. R. China. He received his B.S. in Computer Science from Nanjing University in 2004, and Ph.D. in Computer Science and Engineering from the State University of New York at Buffalo in 2009. He has visited the University of Illinois at Urbana-Champaign (UIUC) as a Post Doc Research Associate. His current research interests include wireless networking and mobile computing, algorithmic network economics, and privacy preservation. He received Excellent Young Scholar award of Shanghai Jiao Tong University in 2011, and Pujiang Scholar award in 2012. He is a member of ACM, CCF, and IEEE. For more information, please visit http://www.cs.sjtu.edu.cn/ fwu/.

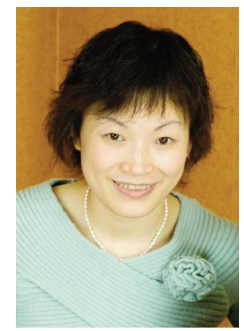

Qian Zhang received the BS, MS, and $\mathrm{PhD}$ degrees from Wuhan University, P. R. China, in 1994, 1996, and 1999 , respectively, all in computer science. She joined Hong Kong University of Science and Technology in Sept. 2005 where she is a full Professor in the Department of Computer Science and Engineering. Before that, she was in Microsoft Research Asia, Beijing, from July 1999, where she was the research manager of the Wireless and Networking Group. Dr. Zhang has published about 300 refereed papers in international leading journals and key conferences in the areas of wireless/Internet multimedia networking, wireless communications and networking, wireless sensor networks, and overlay networking. She is a Fellow of IEEE for contribution to the mobility and spectrum management of wireless networks and mobile communications. Dr. Zhang has received MIT TR100 (MIT Technology Review) worlds top young innovator award. She also received the Best Asia Pacific (AP) Young Researcher Award elected by IEEE Communication Society in year 2004. Her current research is on cognitive and cooperative networks, dynamic spectrum access and management, as well as wireless sensor networks. 